\title{
Urinary biomarkers in pelvic-ureteric junction obstruction: a systematic review
}

\author{
Irene Paraboschi ${ }^{1}$, Guglielmo Mantica ${ }^{2}$, Neil R. Dalton ${ }^{3}$, Charles Turner ${ }^{3}$, Massimo Garriboli ${ }^{4}$ \\ ${ }^{1}$ Paediatric Surgery Unit, Istituto Giannina Gaslini, DiNOGMI, University of Genova, Genoa, Italy; ${ }^{2}$ Department of Urology, Policlinico San \\ Martino Hospital, University of Genova, Genova, Italy; ${ }^{3}$ WellChild Laboratory, Evelina London Children's Hospital, Guy's and St Thomas' National \\ Health Service Foundation Trust, London, UK; ${ }^{4}$ Paediatric Urology, Evelina London Children's Hospital, London, UK \\ Contributions: (I) Conception and design: All authors; (II) Administrative support: I Paraboschi, M Garriboli; (III) Provision of study materials or \\ patients: I Paraboschi, M Garriboli, G Mantica; (IV) Collection and assembly of data: I Paraboschi, M Garriboli, G Mantica; (V) Data analysis and \\ interpretation: I Paraboschi, M Garriboli, G Mantica; (VI) Manuscript writing: All authors; (VII) Final approval of manuscript: All authors. \\ Correspondence to: Guglielmo Mantica, MD. Department of Urology, Policlinico San Martino Hospital, University of Genova, Genova, Italy. \\ Email: guglielmo.mantica@gmail.com.
}

\begin{abstract}
Antenatal hydronephrosis is a common finding detected on prenatal ultrasound. Although hydronephrosis will spontaneously resolve in the majority of newborns, there is a significant amount of cases that will worsen with the risk of a progressive and permanent loss of renal function. There is an increasing concern among experts that the current criteria for evaluation of clinically significant obstructions are limited. Our aim is to provide a systematic review of the available literature on biomarkers of renal injury, potential targets for diagnosis and prognosis of children with hydronephrosis. The main search was conducted in the electronic databases from inception through March 2019 using various combinations of the keywords: pelvicureteric [All Fields] AND junction [All Fields] AND obstruction [All Fields] AND "biomarkers" [MeSH Terms] OR "biomarkers" [All Fields] OR "biomarker" [All Fields]. To broaden the research, additional articles were identified through hand-searching review of the references reported in each study previously selected. Histopathological studies, studies with no control group or with participants suffering from concomitant urological diseases and articles published in language other than English were excluded. Data on study design, sample size, average patient age, hydronephrosis definition used, surgical indication, duration and pattern of follow-up, details on biomarker studied, diagnostic test characteristics, area under the curve (AUC) on receiver operating characteristic (ROC) analysis with the best cut-off (BCO) values, sensitivity, specificity and outcomes were all collected. 38 articles analysing 41 biomarkers were selected. The most frequent proteins investigated were neutrophil gelatinase-associated lipocalin (NGAL) (n=9; 23.7\%), monocyte chemotactic peptide-1 (MCP1) (n=8; 21.1\%), transforming growth factor $\beta 1$ (TGF $\beta 1)$ (n=7; 18.4\%), epidermal growth factor (EGF) (n=6; 15.8\%) and kidney injury molecule 1 (KIM 1) (n=6; 15.8\%). Twenty-seven $(71.1 \%)$ studies evaluated the effect of pyeloplasty on voided urine biomarker concentrations, comparing their values before and after surgery. Twelve (31.6\%) studies investigated the correlation between preoperative biomarker concentration and the anterior posterior renal pelvis diameter (DAP) while 20 (52.6\%) studies investigated the correlation between preoperative biomarker concentration with the split renal function (SRF) measured on nuclear medicine assessments. ROC curves were used to investigate the performance of urinary biomarkers in the total patient data set in $27(71.1 \%)$ studies. Some biomarkers offer promising results. However, a critic analysis of the published studies demonstrates bias and lack of consistency suggesting that larger multicentre and carefully designed prospective studies are still needed to evaluate the clinical usefulness of urinary biomarkers in the diagnosis and follow-up of children with congenital obstructive hydronephrosis.
\end{abstract}

Keywords: Pelvic-ureteric junction obstruction (PUJO); biomarkers; hydronephrosis; kidney injury

Submitted Aug 11, 2019. Accepted for publication Nov 29, 2019.

doi: $10.21037 /$ tau.2020.01.01

View this article at: http://dx.doi.org/10.21037/tau.2020.01.01 


\section{Introduction}

One of the most common causes of renal failure in infants and children is obstructive nephropathy due to congenital hydronephrosis secondary to pelvic-ureteric junction obstruction (PUJO) $(1,2)$. Despite extensive clinical and experimental studies have been undergone over the past decades, fundamental issues regarding the evaluation and management of children with upper urinary tract obstruction remain unsolved.

It is known that the majority of the children with antenatally diagnosed hydronephrosis will have a spontaneous resolution of the dilatation. However, there is a significant amount of cases that will worsen with the risk of a progressive and permanent loss of renal function. The main goal of paediatric urologists is the preservation of kidney function through early selection of patients who will require surgical intervention as opposed to those who have chances to improve spontaneously.

The classic diagnostic tools for investigation of children with upper tract renal obstruction include ultrasound scans, nuclear medicine assessments [(dimercaptosuccinic acid) scan and technetium-99m (Tc-99m DMSA) mercaptoacetyltriglycine (MAG3) renal studies], contrast studies such as micturating cystourethrogram (MCUG) and conventional markers of renal function (such as serum creatinine).

However, these tests are not always adequate predictors for disease progression, particularly for border-line cases. Commonly indication for surgery is currently based on: (I) half-time of the elimination phase (T1/2) of diuretic renogram >15-20 minutes, (II) differential renal function (DRF) less than $40 \%$, (III) deteriorating renal function (more than $5 \%$ in successive radionuclide scans), (IV) progressive thinning of the renal cortex with or without compensatory hypertrophy of the other kidney $(\mathrm{V})$ frequent pyelonephritis (VI) significant grade hydronephrosis, usually greater than $20 \mathrm{~mm}$ (VII) other symptoms such as hypertension, kidney stones, flank pain (3).

However, there is an increasing concern among experts that the aforementioned criteria can't timely identify patients who need surgery from those who can be managed conservatively. The use of urinary biomarkers has been advocated, which could discriminate patients whose renal function will deteriorate from those who will spontaneously improve at an early stage, preventing at the same time unnecessary surgeries. Recently, proteomic has become a very important diagnostic and prognostic tool in the armamentarium of clinicians. Evaluating proteins using proteomic technologies has the ability to increase the understanding and elucidate the cellular and the molecular basis of a particular disorder. In addition, the proteins analysed could be used to identify and quantify a disease at an early stage of occurrence. The etiopathogenesis of renal injury and progression of renal disease in obstructive nephropathy has been well described. It consists of a sequence of cellular and molecular events which includes renal hemodynamic responses, macrophage infiltration of the interstitium, tubular dilatation and apoptosis, accumulation of interstitial fibroblasts through proliferation of resident fibroblasts and epithelial-mesenchymal transformation of renal tubular cells. This process is under the influence of multiple enzymes, cytokines, chemokines, growth factors, signaling molecules and genes (4). Using proteomic analysis, in the last years several attempts have been made to evaluate molecules that could be utilized as potential biomarkers in PUJO in children, most of them concluding to promising results. We have performed an upto-date systematic review of the literature on biomarkers of renal injury and dysfunction, potential targets for diagnosis, prognosis and treatment of children with PUJO.

\section{Methods}

This review was performed according to the Preferred Reporting Items for Systematic Reviews and Metanalysis Statement (http://www.prisma-statement.org/). In order to identify eligible studies, a broad search was conducted in the electronic database MEDLINE from inception through March 2019 using various combinations of keywords such as pelvic-ureteric [All Fields] AND junction [All Fields] AND obstruction [All Fields] AND "biomarkers" [MeSH Terms] OR "biomarkers" [All Fields] OR "biomarker" [All Fields]. To broaden the research, additional records were identified through hand-searching review of the references reported in each article previously selected.

Selection was limited to original articles in English language presenting promising protein biomarkers of PUJO in children. Two independent reviewers (Irene Paraboschi and Massimo Garriboli) performed data extraction and quality assessment. Any disagreement was resolved by consensus or by arbitration of a third author (Guglielmo Mantica) not involved in the initial procedure.

An electronic schedule (Microsoft Excel 2007, Redmond, WA, USA) was pre-established listing authors, article title, journal, year of publication, study period, study design, 
sample size, average age, hydronephrosis definition used, surgical indication, duration and pattern of follow-up, details on biomarker studied, diagnostic test characteristics, area under the curve (AUC) on receiver operating characteristic (ROC) analysis with best cut-off (BCO) values, sensitivity, specificity and outcomes.

The eligibility for the systematic review were studies of any design that reported interleukin 6 (IL6), neutrophil gelatinase-associated lipocalin (NGAL), monocyte chemotactic peptide-1 (MCP1), transforming growth factor $\beta 1$ (TGF $\beta 1$ ), carbohydrate antigen 19.9 (CA19.9), kidney injury molecule 1 (KIM1), glutathione S-transferases (GSTs), antimicrobial peptides (AMPs), such as B defense 1 (BD1), cathelicidin (LL37), hepatocarcinomaintestine-pancreas/pancreatitis-associated protein (HIP/ PAP), and human a defensin 5 (HD5), caspase 3 enzyme, tumor necrosis factor $\alpha(\mathrm{TNF} \alpha)$, thioredoxin (Trx), proximal tubule metallopeptidases (such as CD10, CD13, CD26), intercellular adhesion molecule 1 (ICAM1), lysosomal exoglycosidases (such as HEX A, HEX B, FUC, GAL, MAN, GLU), epidermal growth factor (EGF), emmprin, matrix metalloproteinase 9 (MMP9), tissue inhibitor of metalloproteinase 1 (TIMP1), procollagen III aminoterminal propeptide (PIIINP), regulated on activation, normal $\mathrm{T}$ cell expressed and secreted (RANTES), sFas/Apo-1 (CD95), angiotensinogen (AGT), macrophage inflammatory protein 1a (MIP1a), interferon- $\gamma$-inducible protein-10 (IP10), osteopontin (OPN), cystatin C (CyC), $\beta 2$ microglobulin $(\beta 2 \mathrm{M})$, heme oxygenase 1 (HO1), $\mathrm{N}$-acetyl- $\beta$-D-glucosaminidase (NAG), alkaline phosphatase (ALP), $\gamma$-glutamyl transferase (GGT), endothelin 1 (ET1), semaphorin-3A (SEMA3A), netrin-1, liver-type fatty acidbinding proteins (L-FABP) in urine samples of children (until 18 years old) with unilateral or bilateral congenital PUJO compared to healthy controls or to children with dilated but not obstructed kidneys.

Histopathological studies were excluded from the systematic reviews as well as large scale urinary proteome analyses carried out through liquid chromatography coupled to tandem mass spectrometry (HPLC-MS/ MS) or by mean high throughput techniques as matrice assisted laser desorption ionization or surface enhanced laser desorption ionization mass spectrometry (MALDI/ SELDI-MS). Studies with no control group, studies with participants suffering from concomitant urological diseases and studies published in language other than English were also excluded.

Due to the heterogeneity of studies and especially the very small number of studies referring to the same biomarker a meta-analysis of the available data was deemed unfeasible.

\section{Results}

Out of 79 studies that were initially identified from the main search and through hand-searching review 40 were excluded: 28 were excluded on a title basis, 12 were excluded on abstract basis. Thirty-nine studies were evaluated on a full-text basis. Out of them, 1 had to be excluded due to inappropriate study population age, leaving in the end 38 studies that have been included in this review.

As shown in Prisma flow diagram (Figure 1), 8 articles were excluded because based on large scale urinary proteome analysis, 3 articles because they were written in language other than in English, 11 articles because they only reviewed previous studies, 14 articles because they did not analyse urinary protein biomarkers, 3 articles because they did not refer to paediatric age and 2 articles because they were systematic reviews. Table 1 summarized data of the 38 studies published between November 1997 and October 2018. Out of the 38 studies included in this systematic review, all (100\%) searched for urine biomarkers and $5(13.2 \%)(7,12,18,22,34)$, included also serum samples. Thirty-two (84.2\%) studies considered only patients with unilateral PUJO while $6(15.8 \%)$ included also patients with bilateral obstruction $(5,6,20,21,33,35)$. The quantitative urine protein analysis was performed using commercially available human sandwich enzyme-linked immunosorbent assay (ELISA) kits in 32 (84.2\%) studies. Fourteen (36.8\%) studies compared patients undergoing pyeloplasty [surgical group (SG)] with patients with mild non-obstructive dilatation not requiring surgery [non-surgical group (NSG)] and healthy children [reference group (RG)] $(9,10$, 14-18,20,22,27,29,31-33). Seven (18.4\%) articles analysed urine biomarker concentrations in patients undergoing surgery (SG) and related them with patients conservatively treated (NSG) $(6,19,21,28,36,37,41)$. Thirteen $(34.2 \%)$ studies reported the comparison between surgical patients (SG) and healthy subjects (RG) $(8,11,13,24-26,30,34,35$, $38-40,42)$. Four $(10.5 \%)$ articles compared patients with PUJO with healthy children (RG) without differentiating in the former group those who required surgery $(5,7,12,23)$. Twenty-two (57.9\%) studies evaluated the effect of pyeloplasty on voided urine biomarker concentrations, comparing their values before and after surgery $(9,11$, $14-16,18,22,24,25,27-32,34,35,37-39,41,42)$. Twelve (30.8\%) 


\section{PRISMA 2009 Flow Diagram}
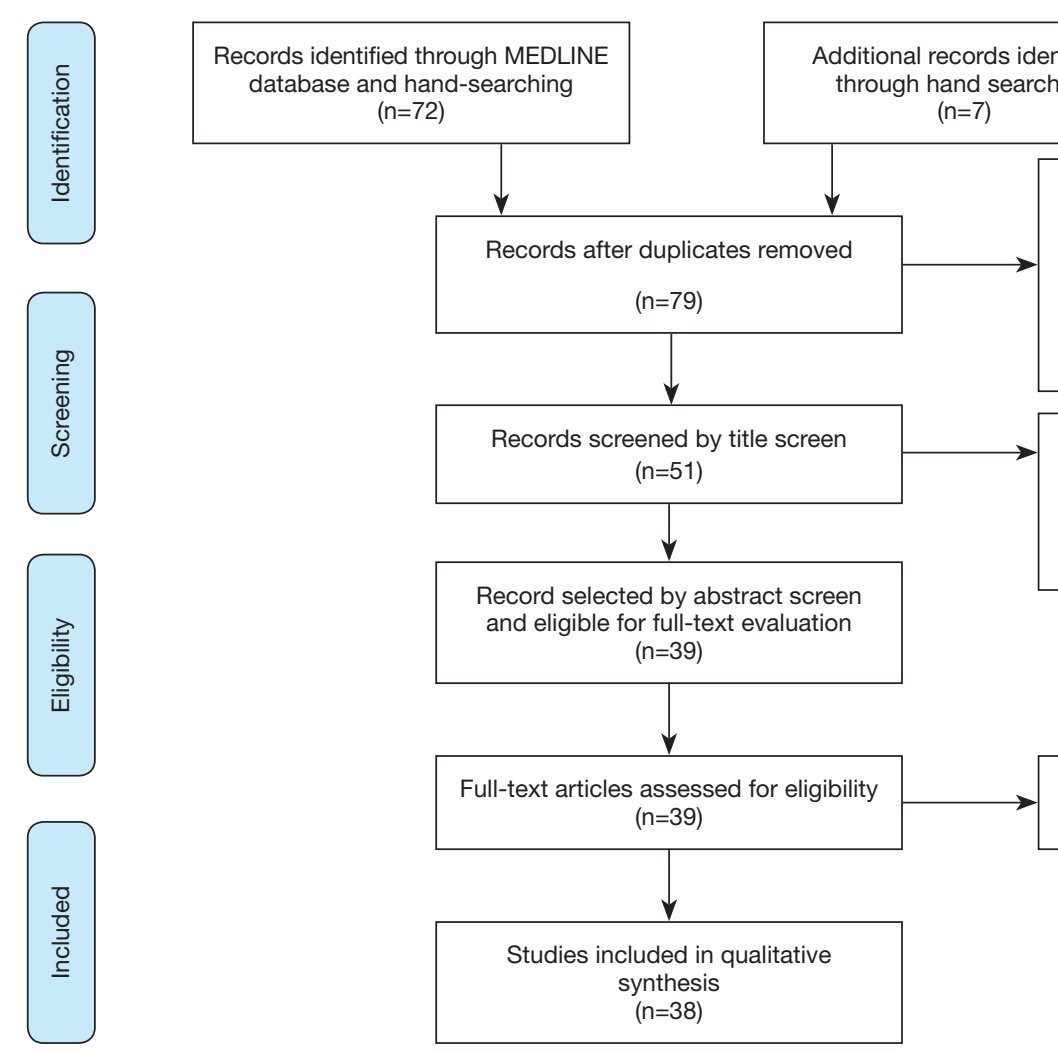

Full-text articles excluded $(n=1)$

- Article not referring to paediatric age $(n=1)$

Figure 1 Prisma flowchart.

studies investigated the correlation between preoperative biomarker concentration with the anterior posterior renal pelvis diameter (DAP) $(9,12,14,16,18,22,23,25,27,30-32)$ while $20(52.6 \%)$ studies investigated the correlation between preoperative biomarker concentration with the split renal function (SRF) evaluated on nuclear medicine assessments $(8,9,12-14,16,18,19,22,25-32,39,41,42)$. ROC curves were used to investigate the performance of urinary biomarkers in the total patient data set in $21(55.3 \%)$ studies (5-9,14-22,24,27-32). Table 2 summarized the results of the 38 articles classified them according to the type of urinary biomarker analysed.

\section{$N G A L$}

Preoperative urinary NGAL levels in surgical PUJO patients significantly differed from healthy controls in nine studies $(5,7,8,13,15,17,26,30,32)$, and from dilated non- obstructed patients in two studies $(17,32)$.

\section{TGFB1}

Preoperative TGF $\beta 1$ levels in surgical PUJO patients were significantly higher than in healthy control group in 3 studies $(23,35,39)$ while Palmer et al. (40) did not identify any significant difference. Preoperative TGF $\beta 1$ levels in surgical PUJO patients were significantly higher than in patients with mild non-obstructive hydronephrosis in the study of El-Sherbiny et al. (41).

A minority of studies performed a ROC analysis to define the diagnostic profile of TGF $\beta 1$. In the series reported by El-Sherbiny et al. (41) mean bladder TGF $\beta 1$ value 3 months after surgery showed a trend towards a decrease, albeit still insignificant. Conversely postoperative mean TGF $\beta 1$ concentration was significantly lower than the preoperative value according to Sager et al. (35) and Taha et al. (39). 
Table 1 Articles on paediatric urinary biomarkers of PUJO

\begin{tabular}{|c|c|c|c|c|c|c|c|c|c|c|c|c|}
\hline Reference & Year & $\begin{array}{l}\text { No. of } \\
\text { participants }\end{array}$ & Age of SG & Laterality & $\begin{array}{l}\text { Urinary } \\
\text { source }\end{array}$ & Biomarker & Method & SG pre op vs. SG post op & SG vs. NSG & SG vs. RG & $\begin{array}{l}\text { Correlation } \\
\text { with APD }\end{array}$ & $\begin{array}{l}\text { Correlation } \\
\text { with SRF }\end{array}$ \\
\hline Yuet al. (5) & 2019 & $\begin{array}{l}\text { PUJO: } n=17 \\
\text { RG: } n=17\end{array}$ & ND & $\begin{array}{l}\text { Unilateral: } \\
\mathrm{n}=12 \text {; bilateral: } \\
\mathrm{n}=5\end{array}$ & Bladder & $\begin{array}{l}\text { IL6; LCN2; MCP1; } \\
\text { TGF } \beta 1\end{array}$ & ELISA & ND & ND & $0.0073 ; 0.0004 ; 0.0005 ; \mathrm{NS}$ & ND & ND \\
\hline $\begin{array}{l}\text { Nabavizadeh } \\
\text { et al. (6) }\end{array}$ & 2019 & $\begin{array}{l}\text { SG early: } \mathrm{n}=34 ; \\
\text { SG delayed: } \\
\mathrm{n}=24 \text {; NSG: } \\
\mathrm{n}=54\end{array}$ & $\begin{array}{l}\text { Mean (standard deviation): } \\
30.0( \pm 8.9) \text { mo }\end{array}$ & $\begin{array}{l}\text { Unilateral: } \\
n=33 ; \text { bilateral: } \\
n=1\end{array}$ & Bladder & CA19.9 & Chemiluminescence assays & ND & $<0.001$ & ND & ND & ND \\
\hline Bieniaś et al. (7) & 2018 & $\begin{array}{l}\text { PUJO: } n=45 \\
\text { RG: } n=21\end{array}$ & $\begin{array}{l}\text { Median [range]: } \\
11.0[2-17] \text { yrs }\end{array}$ & $\begin{array}{l}\text { Unilateral: } \\
\mathrm{n}=45\end{array}$ & Bladder & $\begin{array}{l}\alpha \text {-GST; ח-GST; NGAL; } \\
\text { KIM1 }\end{array}$ & ELISA & ND & ND & ND & ND & ND \\
\hline Gupta et al. (8) & 2018 & $\begin{array}{l}\text { SG: } n=30 ; R G: \\
n=15\end{array}$ & $\begin{array}{l}\text { Mean (range): } \\
4.7(0.3-18.4) \text { yrs }\end{array}$ & $\begin{array}{l}\text { Unilateral: } \\
\mathrm{n}=30\end{array}$ & Bladder & $\begin{array}{l}\text { BD1; NGAL; LL37; } \\
\text { HIP/PAP; HD5 }\end{array}$ & ELISA & ND & ND & $\begin{array}{l}0.0152 ; 0.0009 ; 0.0007 ; 0.0461 ; \\
0.1007\end{array}$ & ND & NS \\
\hline Liet al. (9) & 2018 & $\begin{array}{l}\text { SG: } n=42 ; N S G: \\
n=42 ; R G: n=44\end{array}$ & $\begin{array}{l}\text { Median (range): } \\
2.48(0.17-16.9) \text { yrs }\end{array}$ & $\begin{array}{l}\text { Unilateral: } \\
\mathrm{n}=42\end{array}$ & $\begin{array}{l}\text { Bladder; } \\
\text { pelvis }\end{array}$ & $\begin{array}{l}\text { Semaphorin-3A; } \\
\text { netrin-1 }\end{array}$ & ELISA & $\mathrm{ND} ;<0.01$ & $<0.01 ;<0.01$ & $<0.01 ;<0.01$ & NS; NS & $<0.01 ;<0.01$ \\
\hline $\begin{array}{l}\text { Sadeghi-Bojd } \\
\text { et al. (10) }\end{array}$ & 2018 & $\begin{array}{l}\text { SG: } n=20 ; N S G: \\
n=20 ; R G: n=30\end{array}$ & $\begin{array}{l}\text { Mean (standard deviation): } \\
23.5 \text { ( } \pm 21.79) \text { mo }\end{array}$ & $\begin{array}{l}\text { Unilateral: } \\
\mathrm{n}=20\end{array}$ & Bladder & MCP1 & ELISA & ND & 0.005 & 0.001 & ND & ND \\
\hline Shirazi et al. (11) & 2017 & $\begin{array}{l}\text { SG: } n=31 ; R G: \\
n=33\end{array}$ & $\begin{array}{l}\text { Mean (standard deviation): } \\
22.44( \pm 20.52) \text { mo }\end{array}$ & $\begin{array}{l}\text { Unilateral: } \\
\mathrm{n}=31\end{array}$ & $\begin{array}{l}\text { Bladder; } \\
\text { pelvis }\end{array}$ & TNF $\alpha$; caspase 3 & ELISA & $<0.01 ;<0.01$ & ND & $<0.001 ;<0.001$ & ND & ND \\
\hline Xu et al. (12) & 2017 & $\begin{array}{l}\text { PUJO: } n=156 \\
\text { RG: } n=80\end{array}$ & Median: 2.2 yrs & $\begin{array}{l}\text { Unilateral: } \\
n=156\end{array}$ & Bladder & Trx & ELISA & ND & ND & $<0.001$ & $<0.001$ & $<0.001$ \\
\hline Gerber et al. (13) & 2016 & $\begin{array}{l}\text { SG: } n=12 ; R G: \\
n=12\end{array}$ & $\begin{array}{l}\text { Median [range]: } \\
42 \text { [4-240] mo }\end{array}$ & $\begin{array}{l}\text { Unilateral: } \\
\mathrm{n}=12\end{array}$ & Bladder & $\begin{array}{l}\text { NGAL; KIM1; CD10; } \\
\text { CD13; CD26 }\end{array}$ & ELISA & ND & ND & $0.932 ; 0.799 ; 0.002 ; 0.024 ; 0.007$ & ND & NS \\
\hline $\begin{array}{l}\text { Taranta-Janusz } \\
\text { et al. (14) }\end{array}$ & 2016 & $\begin{array}{l}\text { SG: } n=29 ; \text { NSG: } \\
n=23 ; R G: n=19\end{array}$ & $\begin{array}{l}\text { Median (range): } 1.75 \text { yrs } \\
\text { (1 wk-16.5 yrs) }\end{array}$ & $\begin{array}{l}\text { Unilateral: } \\
\mathrm{n}=29\end{array}$ & Bladder & ICAM1 & ELISA & NS & $<0.05$ & $<0.001$ & $<0.001$ & $<0.05$ \\
\hline Karakus et al. (15) & 2016 & $\begin{array}{l}\text { SG: } n=13 ; N S G: \\
n=14 ; R G: n=9\end{array}$ & $\begin{array}{l}\text { Median [range]: } \\
12[2-56] \mathrm{mo}\end{array}$ & $\begin{array}{l}\text { Unilateral: } \\
\mathrm{n}=13\end{array}$ & Bladder & $\begin{array}{l}\text { NGAL; CyC; IP10; } \\
\text { MCP1; KIM1 }\end{array}$ & ELISA & $\begin{array}{l}\text { Significant; NS; Significant; Significant; } \\
\text { Significant }\end{array}$ & $\begin{array}{l}\text { NS; NS; } 0.038 ; \\
0.037 ; \text { NS }\end{array}$ & $0.032 ; \mathrm{NS} ; 0.024 ; 0.002 ; 0.001$ & ND & ND \\
\hline $\begin{array}{l}\text { Taranta-Janusz } \\
\text { et al. (16) }\end{array}$ & 2015 & $\begin{array}{l}\text { SG: } n=16 ; N S G: \\
n=16 ; R G: n=42\end{array}$ & $4.45 \mathrm{yrs}( \pm 4.66 \mathrm{yrs})$ (mean) & $\begin{array}{l}\text { Unilateral: } \\
\mathrm{n}=16\end{array}$ & Bladder & $\begin{array}{l}\text { HEX; HEX A; HEX B; } \\
\text { FUC; GAL; MAN; GLU }\end{array}$ & $\begin{array}{l}\text { Szajda-modified Marciniak } \\
\text { method }\end{array}$ & $<0.01$ & $\begin{array}{l}\text { NS; <0.01; NS; } \\
\text { NS; NS; NS; NS }\end{array}$ & $\begin{array}{l}<0.01 ;<0.01 ;<0.01 ;<0.01 ;<0.01 ; \\
<0.01 ;<0.01\end{array}$ & NS & NS \\
\hline Noyan et al. (17) & 2015 & $\begin{array}{l}\text { SG: } n=26 ; \text { NSG: } \\
n=36 ; R G: n=20\end{array}$ & $\begin{array}{l}\text { Median [range]: } \\
21[1-48] \mathrm{mo}\end{array}$ & $\begin{array}{l}\text { Unilateral: } \\
\mathrm{n}=26\end{array}$ & Bladder & NGAL; KIM1; FABP & Micro ELISA & ND & $<0.05 ; \mathrm{NS} ; \mathrm{NS}$ & $<0.05 ;$ NS; NS & ND & ND \\
\hline Atar et al. (18) & 2015 & $\begin{array}{l}\text { SG: } n=17 ; \text { NSG: } \\
n=17 ; R G: n=21\end{array}$ & $\begin{array}{l}\text { Mean (standard deviation): } \\
15.3( \pm 14.6) \text { mo }\end{array}$ & $\begin{array}{l}\text { Unilateral: } \\
\mathrm{n}=17\end{array}$ & Bladder & CA19.9 & ELISA & 0.039 & 0.007 & 0.001 & NS & 0.046 \\
\hline Tian et al. (19) & 2015 & $\begin{array}{l}\text { SG: } n=15 \text {; NSG: } \\
n=25\end{array}$ & ND & $\begin{array}{l}\text { Unilateral: } \\
\mathrm{n}=15\end{array}$ & Bladder & $\begin{array}{l}\text { Emmprin; MMP9; } \\
\text { TIMP1 }\end{array}$ & ELISA & ND & $\begin{array}{l}<0.0001 ;<0.05 \\
<0.05\end{array}$ & ND & ND & $\begin{array}{l}\text { NS; 0.0012; } \\
\text { NS }\end{array}$ \\
\hline $\begin{array}{l}\text { Mohammadjjafari } \\
\text { et al. (20) }\end{array}$ & 2014 & $\begin{array}{l}\text { SG: } n=24 ; N S G: \\
n=18 ; R G: n=17\end{array}$ & ND & $\begin{array}{l}\text { Unilateral: } \\
\mathrm{n}=18 \text {; bilateral: } \\
\mathrm{n}=6\end{array}$ & Bladder & EGF; KIM1 & ELISA & ND & $0.016 ; 0.015$ & ND & ND & ND \\
\hline $\begin{array}{l}\text { Mohammadjafari } \\
\text { et al. (21) }\end{array}$ & 2014 & $\begin{array}{l}\text { SG: } n=24 \text {; NSG: } \\
n=18\end{array}$ & Mean: 4.24 mo & $\begin{array}{l}\text { Unilateral: } \\
\mathrm{n}=18 ; \text { bilateral: } \\
\mathrm{n}=6\end{array}$ & Bladder & ET1; MCP1; NAG & ELISA & ND & NS; 0.012; NS & ND & ND & ND \\
\hline Wen et al. (22) & 2014 & $\begin{array}{l}\text { SG: } n=29 ; \text { NSG: } \\
n=30 ; R G: n=30\end{array}$ & $\begin{array}{l}\text { Median (range): } \\
2.2(0.21-17) \text { yrs }\end{array}$ & $\begin{array}{l}\text { Unilateral: } \\
\mathrm{n}=29\end{array}$ & $\begin{array}{l}\text { Bladder; } \\
\text { pelvis }\end{array}$ & PIIINP & ELISA & $<0.01$ & $<0.01$ & $<0.01$ & NS & $<0.05$ \\
\hline
\end{tabular}




\begin{tabular}{|c|c|c|c|c|c|c|c|c|c|c|c|c|}
\hline Reference & Year & $\begin{array}{l}\text { No. of } \\
\text { participants }\end{array}$ & Age of SG & Laterality & $\begin{array}{l}\text { Urinary } \\
\text { source }\end{array}$ & Biomarker & Method & SG pre op vs. SG post op & SG vs. NSG & SG vs. RG & $\begin{array}{l}\text { Correlation } \\
\text { with APD }\end{array}$ & $\begin{array}{l}\text { Correlation } \\
\text { with SRF }\end{array}$ \\
\hline Merrikhi et al. (23) & 2014 & $\begin{array}{l}\text { PUJO: } n=25 \\
\text { RG: } n=25\end{array}$ & $\begin{array}{l}\text { Mean (standard deviation): } \\
7.4( \pm 4.5) \text { yrs }\end{array}$ & $\begin{array}{l}\text { Unilateral: } \\
\mathrm{n}=25\end{array}$ & Bladder & TGF $\beta 1$ & ELISA & ND & ND & 0.001 & NS & ND \\
\hline Madsen et al. (24) & 2013 & $\begin{array}{l}\text { SG: } n=28 ; R G: \\
n=13\end{array}$ & $\begin{array}{l}\text { Median (range): } 8.1 \\
(3.5-15) \text { yrs }\end{array}$ & $\begin{array}{l}\text { Unilateral: } \\
\mathrm{n}=28\end{array}$ & $\begin{array}{l}\text { Bladder; } \\
\text { pelvis }\end{array}$ & $\begin{array}{l}\text { EGF; MCP1; MIP1A; } \\
\text { IP10; CCL5/RANTES }\end{array}$ & ELISA & NS; NS; $0.0001 ; 0.0001 ; 0.0001$ & ND & $\begin{array}{l}0.012 ; 0.005 ; 0.0001 ; 0.1610 ; \\
0.3959\end{array}$ & ND & ND \\
\hline $\begin{array}{l}\text { Gawłowska- } \\
\text { Marciniak et al. } \\
\text { (25) }\end{array}$ & 2013 & $\begin{array}{l}\text { SG: } n=45 ; R G: \\
n=25\end{array}$ & $\begin{array}{l}\text { Mean (standard deviation): } \\
8.4( \pm 5.66) \text { yrs }\end{array}$ & $\begin{array}{l}\text { Unilateral: } \\
\mathrm{n}=45\end{array}$ & $\begin{array}{l}\text { Bladder; } \\
\text { pelvis }\end{array}$ & $\begin{array}{l}\text { TGFB1; CCL5/RANTES; } \\
\text { sFas/Apo-1 }\end{array}$ & ELISA & $\begin{array}{l}<0.05 \text { (younger); }<0.05 \text { (older); }<0.05 \\
\text { (younger); }<0.05 \text { (adolescent); }<0.05 \\
\text { (younger); }<0.05 \text { (older) }\end{array}$ & ND & $\begin{array}{l}<0.05 \text { (older); }<0.05 \text { (adolescent); } ; \\
<0.05 \text { (younger); <0.05 (older); } \\
<0.05 \text { (younger); <0.05 (older); } \\
<0.05 \text { (adolescent) }\end{array}$ & NS & NS \\
\hline Cost et al. (26) & 2013 & $\begin{array}{l}\text { SG: } n=25 ; R G: \\
n=11\end{array}$ & $\begin{array}{l}\text { Median (range): } 1.62 \\
(0.12-18.7) \text { yrs }\end{array}$ & $\begin{array}{l}\text { Unilateral: } \\
\mathrm{n}=25\end{array}$ & $\begin{array}{l}\text { Bladder; } \\
\text { pelvis }\end{array}$ & NGAL & ELISA & ND & ND & 0.004 & ND & 0.004 \\
\hline $\begin{array}{l}\text { Taranta-Janusz } \\
\text { et al. (27) }\end{array}$ & 2013 & $\begin{array}{l}\text { SG: } n=31 \text {; NSG: } \\
n=20 ; R G: n=19\end{array}$ & $\begin{array}{l}\text { Mean (standard deviation): } \\
3.2( \pm 4.3) \text { yrs }\end{array}$ & $\begin{array}{l}\text { Unilateral: } \\
\mathrm{n}=31\end{array}$ & Bladder & AGT & ELISA & NS & $<0.01$ & $<0.01$ & NS & $<0.01$ \\
\hline Li et al. (28) & 2012 & $\begin{array}{l}\text { SG: } n=12 ; \text { NSG: } \\
n=33\end{array}$ & $\begin{array}{l}\text { Median (range): } 8.2 \\
(4.3-18.9) \mathrm{mo}\end{array}$ & $\begin{array}{l}\text { Unilateral: } \\
\mathrm{n}=12\end{array}$ & Bladder & EGF & ELISA & 0.031 & ND & 0.001 & ND & NS \\
\hline $\begin{array}{l}\text { Taranta-Janusz } \\
\text { et al. (29) }\end{array}$ & 2012 & $\begin{array}{l}\text { SG: } n=15 ; \text { NSG: } \\
n=21 ; R G: n=19\end{array}$ & $\begin{array}{l}\text { Median (range): } 0.25 \\
(0.08-8.0 \text { yrs }\end{array}$ & $\begin{array}{l}\text { Unilateral: } \\
\mathrm{n}=15\end{array}$ & $\begin{array}{l}\text { Bladder; } \\
\text { pelvis }\end{array}$ & $\begin{array}{l}\text { MCP1; OPN; CCL5/ } \\
\text { RANTES }\end{array}$ & ELISA & ND; NS; ND & $\begin{array}{l}<0.05 ;<0.05 \\
\text { No }\end{array}$ & $<0.05 ;<0.01 ;$, No & ND & $\begin{array}{l}<0.05 ;<0.05 ; \\
<0.05\end{array}$ \\
\hline Madsen et al. (30) & 2012 & $\begin{array}{l}\text { SG: } n=24 ; R G: \\
n=13\end{array}$ & $\begin{array}{l}\text { Median (range): } 8.0 \\
(3.5-14.5) \text { yrs }\end{array}$ & $\begin{array}{l}\text { Unilateral: } \\
\mathrm{n}=24\end{array}$ & $\begin{array}{l}\text { Bladder; } \\
\text { pelvis }\end{array}$ & NGAL; CyC; $\beta 2 M ;$ OPN & $\begin{array}{l}\text { Bead-based multiplex } \\
\text { sandwich immunoassay }\end{array}$ & NS & ND & NS & NS & NS \\
\hline Liet al. (31) & 2012 & $\begin{array}{l}\text { SG: } n=25 \text {; NSG: } \\
n=25 ; R G: n=30\end{array}$ & $\begin{array}{l}\text { Mean (standard deviation): } \\
2.37( \pm 0.66) \text { yrs }\end{array}$ & $\begin{array}{l}\text { Unilateral: } \\
\mathrm{n}=25\end{array}$ & $\begin{array}{l}\text { Bladder; } \\
\text { pelvis }\end{array}$ & HO1 & ELISA & $<0.01$ & $<0.01$ & $<0.01$ & 0.002 & NS \\
\hline Bartoli et al. (33) & 2011 & $\begin{array}{l}\text { SG: } n=28 ; \text { NSG: } \\
n=48 ; R G: n=30\end{array}$ & ND & $\begin{array}{l}\text { Unilateral: } \\
n=24 ; \text { bilateral: } \\
n=4\end{array}$ & Bladder & EGF; MCP1; $\beta 2 M$ & ELISA & ND & ND & NS; NS; 0.037 & ND & ND \\
\hline $\begin{array}{l}\text { Kajbafzadeh } \\
\text { et al. (34) }\end{array}$ & 2010 & $\begin{array}{l}\text { SG: } n=27 ; R G: \\
n=27\end{array}$ & $\begin{array}{l}\text { Mean (range): } 27.62 \\
(0.5-98) \text { yrs }\end{array}$ & Unilateral & $\begin{array}{l}\text { Bladder; } \\
\text { pelvis }\end{array}$ & CA19.9 & $\begin{array}{l}\text { Electrochemiluminescence } \\
\text { enzyme immunometric kit }\end{array}$ & $<0.001$ & ND & $<0.001$ & ND & ND \\
\hline Sager et al. (35) & 2009 & $\begin{array}{l}\text { SG: } n=19 ; R G: \\
n=19\end{array}$ & $\begin{array}{l}\text { Mean (standard deviation): } \\
6.7 \text { ( } \pm 5.6) \text { yrs }\end{array}$ & $\begin{array}{l}\text { Unilateral: } \\
\mathrm{n}=18 ; \text { bilateral: } \\
\mathrm{n}=1\end{array}$ & $\begin{array}{l}\text { Bladder; } \\
\text { pelvis }\end{array}$ & TGF $\beta 1$ & ELISA & 0.0001 & ND & 0.0001 & ND & ND \\
\hline Shokeir et al. (36) & 2009 & $\begin{array}{l}\text { SG: } n=15 ; \text { NSG: } \\
n=15\end{array}$ & ND & $\begin{array}{l}\text { Unilateral: } \\
\mathrm{n}=15\end{array}$ & Bladder & NAG; ALP; GGT & $\begin{array}{l}\text { Method of Maruhn; Synchron } \\
\text { CX7 system; method of } \\
\text { Bowers and McComb }\end{array}$ & ND & $\begin{array}{l}<0.05 ;<0.05 \\
<0.001\end{array}$ & ND & ND & ND \\
\hline Taha et al. (37) & 2007 & $\begin{array}{l}\text { SG: } n=35 \text {; NSG: } \\
n=15\end{array}$ & $\begin{array}{l}\text { Mean (standard deviation): } \\
5.9( \pm 0.71) \text { yrs }\end{array}$ & $\begin{array}{l}\text { Unilateral: } \\
\mathrm{n}=35\end{array}$ & Bladder & NAG; ALP; GGT & $\begin{array}{l}\text { Method of Maruhn; method of } \\
\text { Bowers and McComb; method } \\
\text { of Szasz }\end{array}$ & $\begin{array}{l}3 \text { mo post op: }<0.05 ; 6 \text { mo post op: } \\
<0.01 ; 6 \text { mo post op: }<0.01\end{array}$ & $\begin{array}{l}<0.001 ;<0.001 \\
<0.001\end{array}$ & ND & ND & ND \\
\hline Taha et al. (38) & 2007 & $\begin{array}{l}\text { SG: } n=35 ; R G: \\
n=10\end{array}$ & $\begin{array}{l}\text { Mean (standard deviation): } \\
5.9( \pm 0.7) \text { yrs }\end{array}$ & $\begin{array}{l}\text { Unilateral: } \\
n=35\end{array}$ & $\begin{array}{l}\text { Bladder; } \\
\text { pelvis }\end{array}$ & ET1 & ELISA & $<0.05$ & ND & Significant & ND & ND \\
\hline
\end{tabular}


Table 1 (continued)

\begin{tabular}{|c|c|c|c|c|c|c|c|c|c|c|c|c|}
\hline Reference & Year & $\begin{array}{l}\text { No. of } \\
\text { participants }\end{array}$ & Age of SG & Laterality & $\begin{array}{l}\text { Urinary } \\
\text { source }\end{array}$ & Biomarker & Method & SG pre op vs. SG post op & SG vs. NSG & SG vs. RG & $\begin{array}{l}\text { Correlation } \\
\text { with APD }\end{array}$ & $\begin{array}{l}\text { Correlation } \\
\text { with SRF }\end{array}$ \\
\hline Taha et al. (39) & 2007 & $\begin{array}{l}\text { SG: } n=35 ; R G: \\
n=30\end{array}$ & $\begin{array}{l}\text { Mean (range): } 5.9(0.5-12) \\
\text { yrs }\end{array}$ & $\begin{array}{l}\text { Unilateral: } \\
n=35\end{array}$ & Bladder & TGFB1; EGF & ELISA & $\begin{array}{l}1 \text { mo post op: <0.05; } 2 \text { mo post op: } \\
<0.05 ; 3 \text { mo post op: NS; } 6 \text { mo post } \\
\text { op: NS; } 9 \text { mo post op: NS; } 12 \text { mo post } \\
\text { op: <0.001; } 1 \text { mo post op: NS; } 2 \text { mo } \\
\text { post op: NS; } 3 \text { mo post op: NS; } 6 \text { mo } \\
\text { post op: Ns; } 9 \text { mo post op: NS; } 12 \text { mo } \\
\text { post op: NS }\end{array}$ & ND; ND & $<0.001 ; \mathrm{NS}$ & $\mathrm{ND} ; \mathrm{ND}$ & NS; ND \\
\hline Palmer et al. (40) & 1997 & $\begin{array}{l}\text { SG: } n=13 ; R G: \\
n=19\end{array}$ & $\begin{array}{l}\text { Mean (range): } 4.6 \text { yrs (1 } \\
\text { mo-11 yrs) }\end{array}$ & $\begin{array}{l}\text { Unilateral: } \\
n=13\end{array}$ & $\begin{array}{l}\text { Bladder; } \\
\text { pelvis }\end{array}$ & TGF $\beta 1$ & ELISA & ND & ND & NS & ND & ND \\
\hline $\begin{array}{l}\text { El-Sherbiny et al. } \\
(41)\end{array}$ & 2002 & $\begin{array}{l}\text { SG: } n=15 \text {; NSG: } \\
n=11\end{array}$ & $\begin{array}{l}\text { Mean (standard deviation): } \\
5.2( \pm 4.7) \text { yrs }\end{array}$ & $\begin{array}{l}\text { Unilateral: } \\
\mathrm{n}=15\end{array}$ & $\begin{array}{l}\text { Bladder; } \\
\text { pelvis }\end{array}$ & TGFB1 & ELISA & NS & $<0.003$ & ND & ND & NS \\
\hline $\begin{array}{l}\text { Grandaliano et al. } \\
\text { (42) }\end{array}$ & 2000 & $\begin{array}{l}\text { SG: } n=24 ; R G: \\
n=15\end{array}$ & $\begin{array}{l}\text { Mean [range]: } 24.4 \text { [2-156] } \\
\text { mo }\end{array}$ & $\begin{array}{l}\text { Unilateral: } \\
n=24\end{array}$ & $\begin{array}{l}\text { Bladder; } \\
\text { pelvis }\end{array}$ & MCP1 EGF & ELISA & $<0.01 ;<0.01$ & ND & $<0.01 ;<0.01$ & ND & $<0.03 ;$ NS \\
\hline
\end{tabular}

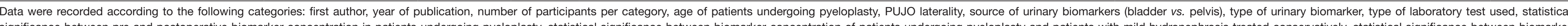

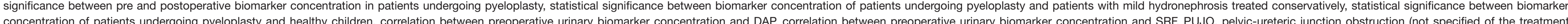

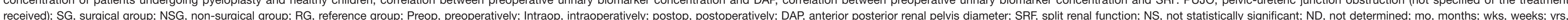

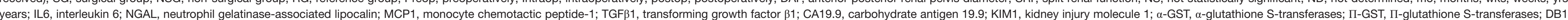

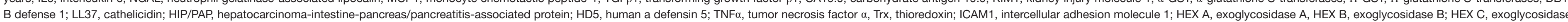

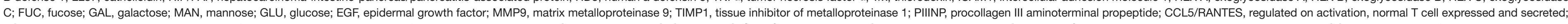

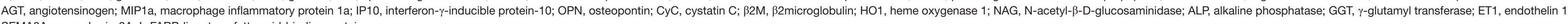
SEMA3A, semaphorin-3A; L-FABP, liver-type fatty acid-binding protein.

\begin{tabular}{|c|c|c|c|c|c|c|c|}
\hline Author & Study groups & Biomarker concentrations & Outcomes & AUC on ROC curve & BCU value & Sensibility & Specificity \\
\hline \multicolumn{8}{|l|}{$\operatorname{NGAL}(n=9 ; 23.7 \%)$} \\
\hline Yuet al. (5) & UPJO: $n=17 ; \mathrm{RG}: n=17$ & ND & $\begin{array}{l}\text { UPJO vs. RG: } 0.0004 \text {; bilateral UPJO vs. unilateral UPJO: }<0.0001 \text {; } \\
\text { bilateral UPJO vs. RG: }<0.0001\end{array}$ & 0.90 & ND & $88 \%$ & $88 \%$ \\
\hline Gupta et al. (8) & $S G: n=30 ; R G: n=15$ & SG: $1.6 \mathrm{ng} / \mathrm{mg}$ Cr (median); RG: $4.9 \mathrm{ng} / \mathrm{mg} \mathrm{Cr}$ & SG vs. RG: 0.0009 & 0.80 & $2.5999 \mathrm{ng} / \mathrm{mg} \mathrm{Cr}$ & $80 \%$ & $76.7 \%$ \\
\hline Gerber et al. (13) & $S G: n=12 ; R G: n=12$ & SG: 902.68/Cr (mean); RG: 66.52/Cr & SG vs. RG: NS & ND & ND & ND & ND \\
\hline Karakus et al. (15) & $\begin{array}{l}\text { SG: } n=13 ; \text { NSG: } n=19 ; \text { RG: } \\
n=9\end{array}$ & ND & $\begin{array}{l}\text { SG vs. RG: } 0.032 ; \text { SG pre op vs. NSG: NS; SG post op } 3 \text { mo vs. SG } \\
\text { pre op: <0.05; SG post op } 6 \text { mo vs. RG: NS }\end{array}$ & 0.85 (95\% Cl: 0.68-1) & $0.688 \mathrm{ng} / \mathrm{mg} \mathrm{Cr}$ & $84.6 \%$ & $77.8 \%$ \\
\hline Noyan et al. (17) & $\begin{array}{l}\text { SG: } n=26 ; \text { NSG: } n=36 ; R G: \\
n=20\end{array}$ & $\begin{array}{l}\text { SG: } 1.8 \mathrm{ng} / \mathrm{mg} \mathrm{Cr} \text { [0-16] (median); NSG: } 0.9 \mathrm{ng} / \mathrm{mg} \mathrm{Cr} \text { [0-21]; RG: } \\
0.5 \mathrm{ng} / \mathrm{mg} \mathrm{Cr}[0-14]\end{array}$ & SG vs. RG: $<0.05$; SG vs. NSG: $<0.05$ & 0.68 (95\% Cl: $0.6-0.7)$ & $0.16 \mathrm{ng} / \mathrm{mg} \mathrm{Cr}$ & $58 \%$ & $75 \%$ \\
\hline Cost et al. (26) & $S G: n=61 ; R G: n=22$ & $\begin{array}{l}\text { SG bladder urine: } 18.6 \mathrm{ng} / \mathrm{mg} \text { (median); SG pelvic urine: } \\
26.2 \mathrm{ng} / \mathrm{mg} ; \mathrm{RG}: 8.3 \mathrm{ng} / \mathrm{mg}\end{array}$ & SG vs. RG: $0.004 ;$ SG pelvic urine vs. SG bladder urine: 0.004 & ND & ND & ND & ND \\
\hline
\end{tabular}




\begin{tabular}{|c|c|c|c|c|c|c|c|}
\hline Author & Study groups & Biomarker concentrations & Outcomes & AUC on ROC curve & $B C U$ value & Sensibility & Specificity \\
\hline Madsen et al. (30) & $S G: n=24 ; R G: n=13$ & 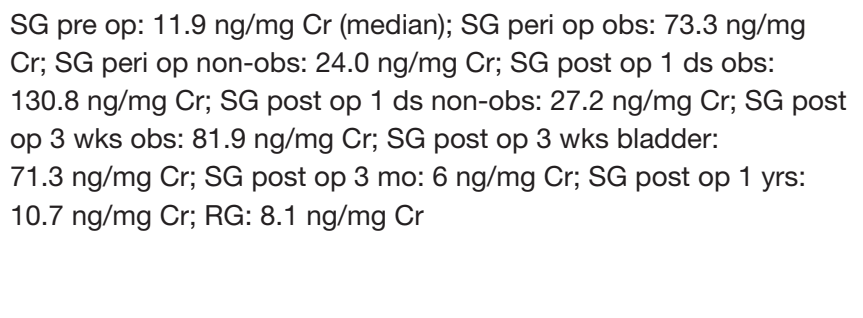 & 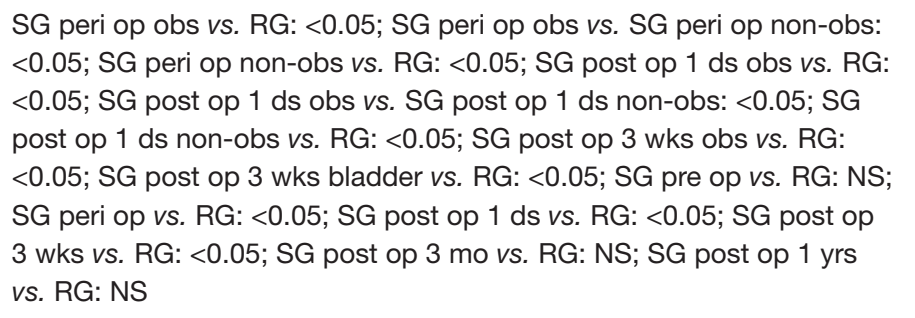 & $0.903(95 \%$ Cl: $0.837-1)$ & $20.57 \mathrm{ng} / \mathrm{mg} \mathrm{Cr}$ & $82 \%$ & $100 \%$ \\
\hline $\begin{array}{l}\text { Wasilewska et al. } \\
\text { (32) }\end{array}$ & $\begin{array}{l}\text { SG: } n=20 ; \text { NSG: } n=20 ; R G: \\
n=25\end{array}$ & $\begin{array}{l}\text { SG pre op: } 23.66 \mathrm{ng} / \mathrm{mg} \text { Cr (median); SG peri op pelvis: } \\
38.48 \text { ng/mg Cr; } \mathrm{SG} \mathrm{post} \mathrm{op} 3 \mathrm{mo}: 5.62 \mathrm{ng} / \mathrm{mg} \text { Cr; NSG: } 3.8 \mathrm{ng} / \mathrm{mg} \\
\text { Cr; RG: } 2.31 \mathrm{ng} / \mathrm{mg} \text { Cr }\end{array}$ & $\begin{array}{l}\text { SG pre op vs. NSG: }<0.05 \text {; SG pre op vs. RG: } 0.01 \text {; SG peri op pelvis } \\
\text { vs. NSG: }<0.01 ; \text { SG peri op pelvis vs. RG: }<0.01 ; \text { SG post op } 3 \text { mo vs. } \\
\text { RG: }<0.05 \text {; NSG vs. RG: }<0.05\end{array}$ & $\begin{array}{l}\text { DRF }<40 \% \text { among SG and NSG: } \\
\text { 0.814 (95\% Cl: } 0.711-0.917) ; \\
\text { DRF <45\% among all patients: } 0.868 \\
\text { (95\% Cl: } 0.796-0.939)\end{array}$ & $\begin{array}{l}2.158 \mathrm{ng} / \mathrm{mg} \mathrm{Cr} ; 4.924 \\
\mathrm{ng} / \mathrm{mg} \mathrm{Cr}\end{array}$ & $100 \% ; 82.4 \%$ & $28.6 \% ; 75.7 \%$ \\
\hline \multicolumn{8}{|l|}{ MCP1 $(n=8 ; 21.1 \%)$} \\
\hline Yu et al. (5) & UPJO: $n=17 ; \mathrm{RG:} n=17$ & ND & $\begin{array}{l}\text { UPJO vs. RG: } 0.0005 \text {; bilateral UPJO vs. unilateral UPJO: }<0.001 \text {; } \\
\text { bilateral UPJO vs. RG: <0.001 }\end{array}$ & 0.89 & ND & ND & ND \\
\hline $\begin{array}{l}\text { Sadeghi-Bojd } \\
\text { et al. (10) }\end{array}$ & $\begin{array}{l}\text { SG: } n=20 ; \text { NSG: } n=20 ; \text { RG: } \\
n=30\end{array}$ & SG: 58.94 ng/L Cr (mean); NSG: 39.58 ng/L Cr; RG: 33.10 ng/L Cr & SG vs. NSG: 0.005; SG vs. RG: 0.001 & ND & ND & ND & ND \\
\hline Karakus et al. (15) & $\begin{array}{l}\text { SG: } n=13 ; \text { NSG: } n=19 ; \text { RG: } \\
n=9\end{array}$ & ND & $\begin{array}{l}\text { SG pre op vs. RG: } 0.002 ; \text { SG pre op vs. NSG: } 0.037 \text {; SG post op } 3 \text { mo } \\
\text { vs. RG: NS }\end{array}$ & 0.93 (95\% Cl: 0.83-1) & $272.3 \mathrm{pg} / \mathrm{mg} \mathrm{Cr}$ & $84.6 \%$ & $88.9 \%$ \\
\hline $\begin{array}{l}\text { Mohammadjafari } \\
\text { et al. (20) }\end{array}$ & $S G: n=24 ; N S G: n=24$ & SG: 1.47 ng/mg Cr (0.86-2.36); NSG: 0.46 ng/mg Cr (0.33-0.81) & SG vs. NSG: 0.012 & 0.723 (95\% Cl: 0.568-0.896) & $0.9270 \mathrm{ng} / \mathrm{mg} \mathrm{Cr}$ & $77 \%$ & $72 \%$ \\
\hline Madsen et al. (24) & SG: $n=28 ; R G: n=13$ & 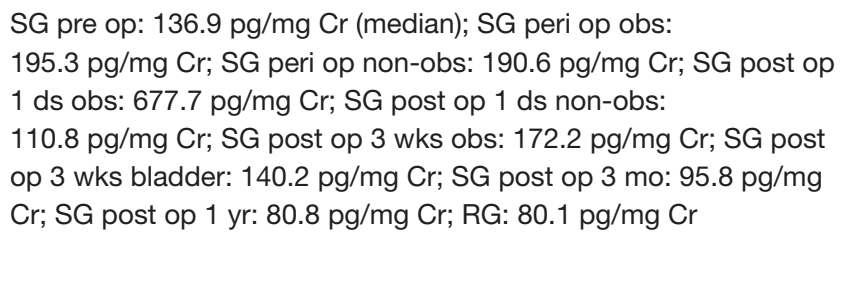 & $\begin{array}{l}\text { SG pre op vs. RG: } 0.005 \text {; SG peri op obs vs. RG: <0.05; SG peri op } \\
\text { obs vs. SG peri op non-obs: <0.05; SG peri op non-obs vs. RG: <0.05; } \\
\text { SG post op } 1 \text { ds obst vs. RG: }<0.05 \text {; SG post op } 1 \text { ds obs vs. SG post } \\
\text { op } 1 \text { ds non-obs: <0.05; SG post op } 3 \text { wks obs vs. RG: <0.05; SG post } \\
\text { op } 3 \text { whs obs vs. SG post op } 3 \text { wks bladder: <0.05; SG post op } 3 \text { mo } \\
\text { vs. RG: NS; SG post op } 1 \text { yr vs. RG: NS; SG pre op vs. SG post op } \\
1 \text { yr: NS }\end{array}$ & 0.78 (95\% Cl: 0.63-0.92) & $93.199 \mathrm{pg} / \mathrm{mg} \mathrm{Cr}$ & $77.8 \%$ & $69.3 \%$ \\
\hline $\begin{array}{l}\text { Taranta-Janusz } \\
\text { et al. (29) }\end{array}$ & $\begin{array}{l}\text { SG: } n=15 ; \text { NSG: } n=21 ; \text { RG: } \\
n=19\end{array}$ & $\begin{array}{l}\text { SG pre op: } 76.77 \mathrm{pg} / \mathrm{mL} \text { pg/mg Cr (median); SG pelvis: } \\
\text { 94.07 pg/mL pg/mg Cr; SG } 3 \text { mo post op: } 56.1 \text { pg/ml pg/mg Cr; } \\
\text { NSG: } 14.04 \mathrm{pg} / \mathrm{mL} \text { pg/mg Cr; RG: } 9.17 \mathrm{pg} / \mathrm{mL} \mathrm{pg} / \mathrm{mg} \mathrm{Cr}\end{array}$ & $\begin{array}{l}\text { SG pre op vs. NSG: }<0.05 \text {; SG pre op vs. RG: <0.05; SG pelvis vs. } \\
\text { NSG: <0.01; SG pelvis vs. RG: <0.01; SG } 3 \text { mo post op vs. NSG: } \\
<0.05 \text {; SG } 3 \text { mo post op vs. RG: }<0.01\end{array}$ & 0.704 (95\% Cl: $0.581-0.827)$ & $0.45 \mathrm{pg} / \mathrm{mg} \mathrm{Cr}$ & $100 \%$ & $0 \%$ \\
\hline Bartoli et al. $(33)^{*}$ & $\begin{array}{l}\text { O-UPJO: } n=12 ; \text { F-UPJO: } \\
n=36 ; \text { OPER: } n=28 ; \text { RG: } \\
n=30\end{array}$ & ND & $\begin{array}{l}\text { OPER vs. O-UPJO: <0.05; OPER vs. F-UPJO: <0.05; O-UPJO vs. RG: } \\
<0.001 \text {; F-UPJO vs. RG: }<0.01\end{array}$ & ND & ND & ND & ND \\
\hline $\begin{array}{l}\text { Grandaliano et al. } \\
\text { (42) }\end{array}$ & SG: $n=24 ; R G: n=15$ & ND & SG pre op vs. RG: $<0.01 ;$ SG pre op vs. SG post op: $<0.01$ & ND & ND & ND & ND \\
\hline \multicolumn{8}{|l|}{ TGFß1 ( $n=7 ; 18.4 \%)$} \\
\hline Yu et al. (5) & UPJO: $n=17 ;$ RG: $n=17$ & ND & $\begin{array}{l}\text { UPJO vs. RG: NS; Bilateral UPJO vs. Unilateral UPJO: NS; Bilateral } \\
\text { UPJO vs. RG: NS }\end{array}$ & 0.56 & ND & ND & ND \\
\hline \multirow[t]{2}{*}{ Merrikhi et al. (23) } & SG: $n=25 ; R G: n=25$ & SG: 87.1 pg/mL (mean) & SG vs. RG: 0.001 & ND & ND & ND & ND \\
\hline & & RG: $14.5 \mathrm{pg} / \mathrm{mL}$ & & & & & \\
\hline
\end{tabular}


Table 2 (continued)

\section{Study groups}

G: $n=45$ (young children:

Marciniak et

(25) $\mathrm{n}=25$ (young children: $\mathrm{n}=5$; (a) $n=15$ )

Sager et al. (35)

SG: $n=19 ;: R G: n=19$

Taha et al. (39)

SG: $n=35 ; \mathrm{RG}: \mathrm{n}=30$

Palmer et al. (40) SG: $n=13 ; \mathrm{RG}: \mathrm{n}=19$

y et al. SG: $n=15$; NSG: $n=11$

$\operatorname{EGF}(n=6 ; 15.8 \%)$

Mohammadjafari $\quad$ SG: $n=24$; NSG: $n=18$; RG:

et al. (21)

Madsen et al. (24) SG: $n=28: R G: n=13$

\section{Lietal. (28) SG: $n=12$ : NSG: $n=33$}

Bartoli et al. (33)" O-UPJO: n=12; F-UPJO: $n=36 ;$ OPER: $n=28$; RG: $\mathrm{n}=30$

\section{Biomarker concentrations}

children $4.495 .18 \mathrm{pg} / \mathrm{mg} \mathrm{Cr}$ (mean); SG pelvic urine older children $3,869.00 \mathrm{pg} / \mathrm{mg}$ Cr; SG pelvic uring adolescents 1,283.54 pg/mg Cr; SG bladder urine young

children 4,652.33 pg/mg Cr (mean); SG bladder urine older children 1,727.09 pg/mg Cr; SG bladder urine adolescents $854.44 \mathrm{pg} / \mathrm{mg}$ Cr; RG bladder urine young children 4,111.71 pg/mg Cr (mean); $\mathrm{RG}$ bladder urine older $\mathrm{klldren} 1,370.29 \mathrm{pg} / \mathrm{mg} \mathrm{Cr}$, RG bladder urine $12 \mathrm{mo}$ post op $595.44 \mathrm{pg} / \mathrm{mg} \mathrm{Cr}$ (mean) $\mathrm{SG}$ bledder wine older children 12 mo post op $60284 \mathrm{pg} / \mathrm{mg} \mathrm{Cr}$. SG bladder urine adolescents children 12 mo post op $450.96 \mathrm{pg} / \mathrm{mg} \mathrm{Cr}$

SG pre op: $92.5 \mathrm{pg} / \mathrm{mL}$ (mean); RG: $35.8 \mathrm{pg} / \mathrm{mL}$; SG peri op pelvis: $223 \mathrm{pg} / \mathrm{mL}$. SG post op: $48.7 \mathrm{pg} / \mathrm{mL}$

SG pre op: $374 \mathrm{pg} / \mathrm{mg} \mathrm{Cr}$; SG post op 1 yr: $157 \mathrm{pg} / \mathrm{mg} \mathrm{Cr}$; SG p op < 1 yr: 601 pg/mg Cr; SG pre op > 1 yr: 307 pg/mg Cr; RG:

SG bladder: $31.2 \mathrm{pg} / \mathrm{mL}$ (mean): SG pelvis: $82.4 \mathrm{pg} / \mathrm{mL}$; RG $26.6 \mathrm{pg} / \mathrm{mL}$

SG bladder pre op: $68 \mathrm{pg} / \mathrm{mg} \mathrm{Cr}$ (mean); SG pelvis per op: 285 pg/mg Cr; SG bladder post op 3 mo: 39 pg/mg Cr; NSG bl

SG: 21.07 ng/mg Cr (median); NSG: 12.86 ng/mg Cr; RG: ND

SG pre op: $7.4 \mathrm{ng} / \mathrm{mg}$ Cr (median); SG peri op obs: $10.4 \mathrm{ng} / \mathrm{mg} \mathrm{Cr}$ SG peri op non-obs: $10.9 \mathrm{ng} / \mathrm{mg}$ Cr; SG post op 1 ds obs:

$12.9 \mathrm{ng} / \mathrm{mg} \mathrm{Cr} ;$ SG post op $1 \mathrm{ds}$ non-obs: $5.6 \mathrm{ng} / \mathrm{mg} \mathrm{Cr}$ : SG post op 3 wks obs: $9.5 \mathrm{ng} / \mathrm{mg}$ Cr: SG post op 3 wks bladder: $7.2 \mathrm{ng} / \mathrm{mg}$ Cr: SG post op 3 mo: $4.6 \mathrm{ng} / \mathrm{mg} \mathrm{Cr}$; SG post op 1 yr: $3.5 \mathrm{ng} / \mathrm{mg}$ Cr $\mathrm{RG}: 4.0 \mathrm{ng} / \mathrm{mg} \mathrm{Cr}$

SG <6 mo post birth: $38 \mathrm{ng} / \mathrm{mg} \mathrm{Cr}$ (median); SG pre op: 46; SG 3-12 mo post op: $55 \mathrm{ng} / \mathrm{mg}$ Cr; NSG <6 mo post birth: $50 \mathrm{ng} / \mathrm{mg}$ Cr: NSG 6-12 mo post birth: 59 ng/mg Cr: NSG >12 mo post birth $69.5 \mathrm{ng} / \mathrm{mg} \mathrm{Cr}$

\section{Outcomes}

Ge older children vs. RG bladder urine older children: 0.05 ; SG pelvic urine adolescents vs. RG bladder urine adolescents: 0.001 ; SG bladder urine young children SG, SG bladder urine

adolescent: $<0.001 ;$ SG bladder urine young children vs. SG bladder urine older children: $<0.001$; SG bladder urine young children pre op vo. Sa be

SG pre op vs. RG: $0.0001 ;$ SG peri op pelvis vs. SG pre op: $<0.05$; SG post op vs. SG pre op: 0.000

SG pre op vs. RG: <0.001; SG post op 1 mo vs. SG pre op: <0.05; SG post op 1 mo vs. RG: <0.001; SG post op 2 mo vs. SG pre op: <0.05; SG post op 2 mo vs. RG: $<0.001$; SG post op 3 mo vs. SG pre op: NS; SG post op 3 movs. RG: $<0.001$; SG post op 6 mo vs. SG pre op: NS; SG post op 9 mo vs. RG: <0 001: SG post op 12 movs SG pre op: $<0.001$. SG post op 12 mo vs. RG: NS. SG pre op < 1 yr vs. SG pre op $>1$ yr: $<0.01$

SG pelvis $v$ s. SG urine: 0.03 . SG bladder $v$ s. RG: NS

AUC on ROC curve

CU value

SG pre op vs. NSG: $<0.003$; SG post op 3 mo vs. SG pre op: NS; SG bladder < 1 yr vs. SG bladder > 1 yr: NS; NSG bladder < 1 yr vs. NSG $S G$ pelvis peri op $>1$ yr:

SG vs. NSG: 0.016

ND

90 pg/mg Cr; <1 yr: $330 \quad 100 \% ; 100 \% ; \quad 80 \% ; 100 \%$ $\mathrm{pg} / \mathrm{mg} \mathrm{Cr} ;>1 \mathrm{yr}: 200 \mathrm{pg} / \quad 100 \%$

SG pre op vs. RG: 0.012 ; SG peri op obs vs. RG: $<0.05$; $S G$ peri op non-obs vs. RG: $<0.05$; SG post op 1 ds obs vs. RG: $<0.05$; SG post non-obs vs. RG: $<0.05$; SG post op 1 ds obs vs. RG: $<0.05$; SG post 1 ds obs vs. SG post op 1 ds non-obs: $<0.05$; SG post op 3 wks obs vs. RG: $<0.05$; SG post op 3 wks obs vs. SG post op 3 wks bladder: <0.05; SG post op 3 mo vs. RG: NS; SG post op 1 yr vs. RG: NS; SG pre op vs. SG post op 1 yr: NS

NSG $<6$ mo post birth vs. NSG 6-12 mo post birth 0 0.015: NSG $<6$ mo 0.789 post birth VS. NSG $>12$ mo post birth: $<0.01$ : NSG 6-12 mo post bith vs. NSG $>12$ mo post birth: $0.017 ;$ SG $<6$ mo post birth vs. SG $3-12$ mo post op: $<0.01 ;$ SG $<6$ mo post birth vs. SG pre op: NS; NSG $<6$ mo post birth vs. $S G<6$ mo post birth: 0.001

O-UPJO vs. RG: <0.05: O-UPJO vs. F-UPJO: $<0.05$
$29 \mathrm{pg} / \mathrm{mg} \mathrm{C}$

0.728 (95\% Cl: $0.560-0.896)$

$16.855 \mathrm{ng} / \mathrm{mg} \mathrm{Cr}$

$71 \%$

0.75 (95\% Cl: $0.60-0.90)$

$4.71 \mathrm{ng} / \mathrm{mg} \mathrm{Cr}$

$69.2 \%$ 
Table 2 (continued)






\begin{tabular}{|c|c|c|c|c|c|c|c|}
\hline Author & Study groups & Biomarker concentrations & Outcomes & AUC on ROC curve & $\mathrm{BCU}$ value & Sensibility & Specificity \\
\hline $\begin{array}{l}\text { Taranta-Janusz } \\
\text { et al. (29) }\end{array}$ & $\begin{array}{l}\text { SG: } n=15 ; \text { NSG: } n=21 ; \text { RG: } \\
n=19\end{array}$ & $\begin{array}{l}\text { SG pre op: } 21.29 \text { pg/mL pg } / \mathrm{mg} \text { Cr (median); SG pelvis: } 45.42 \mathrm{pg} / \\
\mathrm{mL} \text { pg/mg Cr; SG } 3 \text { mo post op: } 31.11 \mathrm{pg} / \mathrm{mL} \mathrm{pg} / \mathrm{mg} \mathrm{Cr} \text { NSG: } \\
35.33 \mathrm{pg} / \mathrm{mL} \mathrm{pg} / \mathrm{mg} \text { Cr; RG: } 13.2 \mathrm{pg} / \mathrm{mL} \mathrm{pg} / \mathrm{mg} \mathrm{Cr}\end{array}$ & $\begin{array}{l}\text { SG pelvis vs. NSG: }<0.01 \text {; SG pelvis vs. RG: }<0.01 \text {; SG } 3 \text { mo post op } \\
\text { vs. NSG: }<0.05 \text {; } \mathrm{SG} 3 \text { mo post op vs. RG: }<0.01\end{array}$ & 0.693 (95\% Cl: 0.58-0.807) & $0.865 \mathrm{pg} / \mathrm{mg} \mathrm{Cr}$ & $100 \%$ & $0 \%$ \\
\hline \multicolumn{8}{|l|}{ CA19.9 $(n=3 ; 7.9 \%)$} \\
\hline $\begin{array}{l}\text { Nabavizadeh } \\
\text { et al. (6) }\end{array}$ & $\begin{array}{l}\text { SG delayed: SG: } n=24 ; \text { SG } \\
\text { early: } n=34 ; \text { NSG: } n=54\end{array}$ & $\begin{array}{l}\text { NSG: } 37.83 \mathrm{U} / \mathrm{mL} \text { (mean); SG delayed: } 145.45 \mathrm{U} / \mathrm{mL} ; \text { SG early: } \\
244.62 \mathrm{U} / \mathrm{mL}\end{array}$ & NSG vs. SG delayed vs. SG early: $<0.001 ;$ NSG vs. SG delayed: $<0.07$ & $0.87(95 \%$ Cl $0.79-0.94)$ & $52.6 \mathrm{U} / \mathrm{mL}$ & $92.0 \%$ & $70.9 \%$ \\
\hline Atar et al. (18) & $\begin{array}{l}\text { SG: } n=17 ; \text { NSG: } n=17 \text {; RG: } \\
n=21\end{array}$ & $\begin{array}{l}\text { SG: } 143 \mathrm{U} / \mathrm{mL} \text { (mean); NSG: } 67.8 \mathrm{U} / \mathrm{mL} \text {; RG: } 13.2 \mathrm{U} / \mathrm{mL} \text {; SG } 3 \mathrm{mo} \\
\text { post op: } 55 \mathrm{U} / \mathrm{mL}\end{array}$ & $\begin{array}{l}\text { SG vs. NSG: } 0.007 \text {; SG vs. RG: } 0.001 \text {; NSG vs. RG: NS; SG vs. SG } 3 \\
\text { mo post op: } 0.039\end{array}$ & $\begin{array}{l}\text { SG vs. NSG: } 0.8 \text { ( } 95 \% \text { Cl: } 0.634- \\
\text { 0.981); Obs vs. non-obs: } 0.8\end{array}$ & $15.7 \mathrm{U} / \mathrm{mL} ; 85.5 \mathrm{U} / \mathrm{mL}$ & $100 \% ; 76 \%$ & $21 \% ; 85 \%$ \\
\hline $\begin{array}{l}\text { Kajbafzadeh } \\
\text { et al. (34) }\end{array}$ & SG: $n=27 ; R G: n=27$ & $\begin{array}{l}\text { SG pre op: } 319.20 \mathrm{U} / \mathrm{mL} \text { (mean); SG post op } 3 \mathrm{mo:} 53.82 \mathrm{U} / \mathrm{mL} \text {; SG } \\
\text { post op } 9 \mathrm{mo}: 18.40 \mathrm{U} / \mathrm{mL} \text {; SG peri op pelvis: } 1,765.67 \mathrm{U} / \mathrm{mL} \text {; RG: } \\
16.91 \mathrm{U} / \mathrm{mL}\end{array}$ & $\begin{array}{l}\text { SG pre op vs. RG: }<0.001 \text {; SG post op } 3 \text { mo op vs. RG: }<0.001 \text {; SG } \\
\text { post op } 3 \text { mo vs. SG pre op: }<0.001 \text {; SG post op } 9 \text { mo vs. SG pre op: } \\
<0.001 \text {; SG peri op pelvis vs. SG pre op: <0.001 }\end{array}$ & ND & $30.6 \mathrm{U} / \mathrm{mL}$ & $100 \%$ & $82.6 \%$ \\
\hline \multicolumn{8}{|l|}{ NAG $(n=3 ; 7.9 \%)$} \\
\hline $\begin{array}{l}\text { Mohammadjafari } \\
\text { et al. (20) }\end{array}$ & SG: $n=24 ;$ NSG: $n=24$ & SG: $1.36 \mathrm{IU} / \mathrm{mg}$ Cr (0.77-2.74); NSG: $0.89 \mathrm{IU} / \mathrm{mg} \mathrm{Cr}(0.49-1.71)$ & SG vs. NSG: NS & 0.627 (95\% Cl: $0.5-0.844)$ & $1.1913 \mathrm{IU} / \mathrm{mg} \mathrm{Cr}$ & $62 \%$ & $67 \%$ \\
\hline Shokeir et al. (36) & SG: $n=15 ; N S G: n=15$ & SG: $8.34 \mathrm{mU} / \mathrm{mg} \mathrm{Cr}$ (mean); NSG: $6.91 \mathrm{mU} / \mathrm{mg} \mathrm{Cr}$ & SG vs. NSG: $<0.05$ & ND & ND & ND & ND \\
\hline Taha et al. (37) & SG: $n=35 ;$ NSG: $n=15$ & 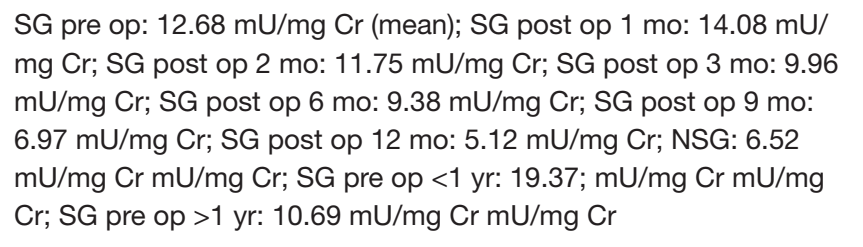 & $\begin{array}{l}\text { SG vs. NSG: }<0.001 \text {; SG post op } 3 \text { mo vs. SG pre op: }<0.05 \text {; SG pre op } \\
<1 \text { yr vs. SG pre op >1 yr: significant }\end{array}$ & ND & $7.8 \mathrm{mU} / \mathrm{mg} \mathrm{Cr}$ & $97.1 \%$ & $80 \%$ \\
\hline \multicolumn{8}{|l|}{$\operatorname{ALP}(\mathrm{n}=2 ; 5.3 \%)$} \\
\hline \multirow[t]{2}{*}{ Shokeir et al. (36) } & SG: $n=15 ; N S G: n=15$ & SG: $40.35 \mathrm{UI/g}$ Cr (mean) & SG vs. NSG: $<0.05$ & ND & ND & ND & ND \\
\hline & & NSG: $28.74 \mathrm{UI} / \mathrm{g} \mathrm{Cr}$ & & & & & \\
\hline Taha et al. (37) & SG: $n=35 ;$ NSG: $n=15$ & 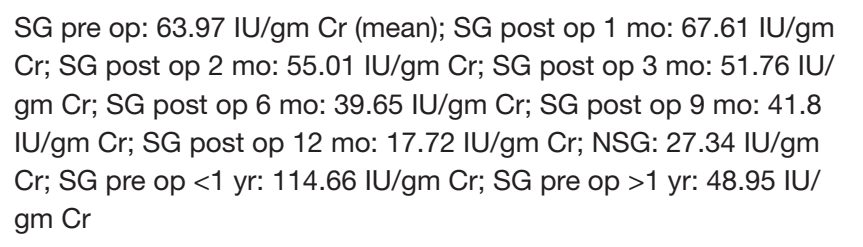 & $\begin{array}{l}\text { SG pre op vs. NSG: }<0.001 ; \text { SG post op } 6 \text { mo vs. SG pre op: }<0.01 ; \text { SG } \\
\text { pre op }<1 \text { yr vs. SG pre op }>1 \text { yr: significant }\end{array}$ & ND & $34.5 \mathrm{IU} / \mathrm{gm} \mathrm{Cr}$ & $91.4 \%$ & $100 \%$ \\
\hline \multicolumn{8}{|l|}{ GGT $(n=2 ; 5.3 \%)$} \\
\hline Shokeir et al. (36) & SG: $n=15 ;$ NSG: $n=15$ & SG: $49.54 \mathrm{Ul} / \mathrm{g} \mathrm{Cr}$ (mean); NSG: $57.21 \mathrm{Ul} / \mathrm{g} \mathrm{Cr}$ & SG vs. NSG: $<0.001$ & ND & ND & ND & ND \\
\hline Taha et al. (37) & SG: $n=35$; NSG: n=15 & 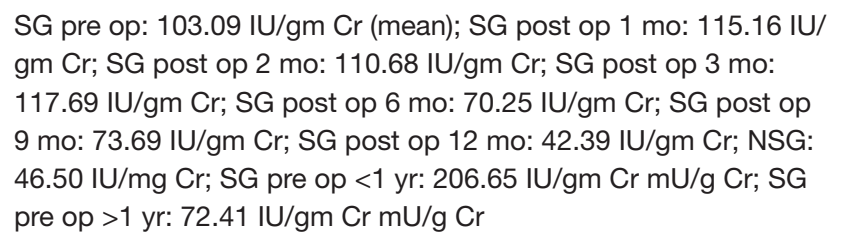 & $\begin{array}{l}\text { SG pre op vs. NSG: }<0.001 ; \text { SG post op } 6 \text { mo vs. SG pre op: }<0.01 ; \text { SG } \\
\text { pre op }<1 \text { yr vs. SG pre op }>1 \text { yr: significant }\end{array}$ & ND & $54 \mathrm{IU} / \mathrm{gm} \mathrm{Cr}$ & $62.9 \%$ & $100 \%$ \\
\hline \multicolumn{8}{|l|}{ IP10 (n=2; 5.3\%) } \\
\hline Karakus et al. (15) & $\begin{array}{l}\text { SG: } n=13 ; \text { NSG: } n=14 ; \text { RG: } \\
n=9\end{array}$ & ND & $\begin{array}{l}\text { SG pre op vs. NSG: } 0.038 \text {; SG pre op vs. RG: } 0.024 \text {; SG pre op vs. SG } \\
\text { post op } 3 \text { yrs: NS }\end{array}$ & 0.68 (95\% Cl: $0.44-0.92)$ & $298.8 \mathrm{pg} / \mathrm{mg} \mathrm{Cr}$ & $61.5 \%$ & $88.9 \%$ \\
\hline
\end{tabular}

Table 2 (continued) 
Table 2 (continued)

Author Study groups

Biomarker concentrations

Outcomes

AUC on ROC curve

$B C U$ value

SG pre op vs. RG: NS; SG peri op vs. RG: $<0.05$; SG peri op obs vs.

op 3 movs. RG: NS: SG post op 1 ys vs. RG: NS

$\mathrm{ET1}(\mathrm{n}=2 ; 5.3 \%)$

Mohammadjafari SG: $n=24:$ NSG: $n=24$

et al. (20)

Taha et al. (38) SG: $n=35 ; \mathrm{RG}: \mathrm{n}=10$

$\beta 2 \mathrm{M}(\mathrm{n}=2 ; 5.3 \%)$

Madsen et al. (30) SG: $n=24 ; R G: n=13$

Bartoli et al. (33) O-UPJO: n=12; F-UPJO: =36; OPER: $\mathrm{n}=28$; $\mathrm{RG}$

SG pre op: $0.88 \mathrm{ng} / \mathrm{mg} \mathrm{Cr}(0.55-1.23)$; NSG pre op: $0.46 \mathrm{ng} / \mathrm{mg} \mathrm{Cr}$ $(0.33-0.81)$

SG pre op: $6.56 \mathrm{fmol} / \mathrm{mg} \mathrm{Cr}$ (mean); SG peri op pelvis:

18.18 mol/mg Cr; SG post op $1 \mathrm{mo:} 11.95 \mathrm{fmol} / \mathrm{mg} \mathrm{Cr}$; SG post

SG pre op vs. NSG pre op: NS

0.657 (95\% Cl: 0.473-0.841)

$0.7509 \mathrm{ng} / \mathrm{mg} \mathrm{Cr}$

$75 \%$

SG peri op pelvis vs. SG pre op: <0.001; SG post op 1 mo vs. SG post

op 1 mo: <0.001; SG post op $2 \mathrm{mo}$ vs. SG post op $2 \mathrm{mo}:<0.001$; $S G$

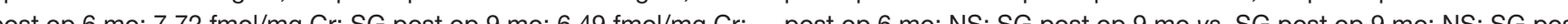

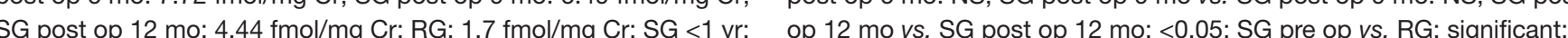

$12.37 \mathrm{fmol} / \mathrm{mg} \mathrm{Cr} ; \mathrm{SG}>1$ yr: $4.84 \mathrm{fmol} / \mathrm{mg}$ Cr

$S G<1$ yr vs. $S G>1$ yr: $<0.001$

SG pre op: $109.8 \mathrm{ng} / \mathrm{mg} \mathrm{Cr}$ (median); SG peri op obs: $207.5 \mathrm{ng} /$ $\mathrm{mg}$ Cr; SG peri op non-obs: $172.5 \mathrm{ng} / \mathrm{mg} \mathrm{Cr}$; SG post op 1 ds obs: $267.6 \mathrm{ng} / \mathrm{mg} \mathrm{Cr} ;$ SG post op 1 ds non-obs: $81.8 \mathrm{ng} / \mathrm{mg} \mathrm{Cr}$; SG pos op 3 whs obs. $152.4 \mathrm{ng} / \mathrm{mg}$ Cr, SG post op 3 whs bladder. 131.7$$
\mathrm{ND}
$$

SG peri op obs vs. RG: <0.05; SG peri op non-obs vs. RG: $<0.05$; SG post op 1 ds obs vs. RG. $<0.05$, SG post op 1 ds obs vs. SG post op

05: SG post op 1 ds $Y s . R G:<0.05$

OPER vs. RG: <0.05; OPER vs. F-UPJO: <0.01; OPER vS. 0-UPJO: 01: O-UPJO vs. RG: $<0.01$; F-UPJO vs. RG: $<0.05$

SG vs. RG: NS; SG vs. NSG: NS; NSG vs. RG: 0.004; SG pre op vs. SG ND post op: NS

SG: $n=13$; NSG: $n=19$; RG:

Karakus et al. (15) $\quad S G=0$

Madsen et al. (30) SG: $n=24: R G: n=13$

SG pre op: 99.0 ng/mg Cr (median); SG peri op obs: $122.3 \mathrm{ng} / \mathrm{mg}$ Cr; SG peri op non-obs: $116.2 \mathrm{ng} / \mathrm{mg}$ Cr; SG post op 1 ds obs: op 3 wks obs: $111.5 \mathrm{ng} / \mathrm{mg}$ Cr; SG post op 3 wks bladder: 1102 $\mathrm{ng} / \mathrm{mg} \mathrm{Cr}$; SG post op $3 \mathrm{mo:} 85.2 \mathrm{ng} / \mathrm{mg} \mathrm{Cr}$; SG post op 1 yr: 74.2 $\mathrm{ng} / \mathrm{mg} \mathrm{Cr} ; \mathrm{RG}: 99.5 \mathrm{ng} / \mathrm{mg} \mathrm{Cr}$

OPN $(n=2 ; 5.3 \%)$

Taranta-Janusz et $\quad$ SG: $n=15$; NSG: $n=21$; RG: al. (29)

SG pre op: $52.29 \mathrm{ng} / \mathrm{mL} \mathrm{ng} / \mathrm{mg} \mathrm{Cr}$ (median); SG pelvis: $73.1 \mathrm{ng} / \mathrm{m}$ $\mathrm{ng} / \mathrm{mg} \mathrm{Cr}$; SG 3 mo post op: $80.52 \mathrm{ng} / \mathrm{mL} \mathrm{ng} / \mathrm{mg} \mathrm{Cr}$; NSG $19.37 \mathrm{ng} / \mathrm{mL} \mathrm{ng} / \mathrm{mg}$ Cr; RG: $39.32 \mathrm{ng} / \mathrm{mL} \mathrm{ng} / \mathrm{mg}$ Cr

SG pre op: 1,664.9 ng/mg Cr (median); SG peri op obs: $1,218.9 \mathrm{ng} / \mathrm{mg}$ Cr; SG peri op non-obs: $1,439.3 \mathrm{ng} / \mathrm{mg}$ Cr; SG post
op $1 \mathrm{ds}$ obs: $2,131.7 \mathrm{ng} / \mathrm{mg}$ Cr; SG post op $1 \mathrm{ds}$ non-obs: 769.0 op $1 \mathrm{ds}$ obs: $2,131.7 \mathrm{ng} / \mathrm{mg} \mathrm{Cr}$; SG post op $1 \mathrm{ds}$ non-obs: 769.0
$\mathrm{ng} / \mathrm{mg}$ Cr; SG post op 3 wks obs: $1,282.0 \mathrm{ng} / \mathrm{mg} \mathrm{Cr}$; SG post op $\mathrm{ng} / \mathrm{mg} \mathrm{Cr}$; SG post op 3 wks obs: $1,282.0 \mathrm{ng} / \mathrm{mg} \mathrm{Cr}$; SG post op
3 wks bladder: $1,168.8 \mathrm{ng} / \mathrm{mg} \mathrm{Cr}$ SG post op $3 \mathrm{mo:} 1,295.9 \mathrm{ng} / \mathrm{mg}$ $\mathrm{Cr}$; SG post op $1 \mathrm{yr}: 1,409.4 \mathrm{ng} / \mathrm{mg} \mathrm{Cr}$; RG: $1,139.7 \mathrm{ng} / \mathrm{mg} \mathrm{Cr}$

SG periop obs vs RG: <0.05: SG post op 1 ds obs vs RG: $<0.05$; SG postop 1 ds obs vs. SG post op 1 ds non-obs: $<0.05$

$3 \mathrm{fmol} / \mathrm{mg} \mathrm{Cr} ; 4 \mathrm{fmol} / \mathrm{m}$ $\operatorname{Cr}(\mathrm{SG}<1 \mathrm{yr}) ; 2.5$

$7.3 \% ; 100 \%$ $81.5 \%$

Madsen et al. (30) SG: $\mathrm{n}=24$; $\mathrm{RG}: \mathrm{n}=13$
SG pelvis vs. NSG: $<0.01$; SG pelvis vs. RG: $<0.01$; SG 3 mo post op $\quad 0.666(95 \%$ Cl: $0.544-0.787)$ vs. NSG: $<0.01$; SG 3 mo post op vs. RG: $<0.01$

SG post op 1 ds obs vs. RG: <0.05; SG post op 1 ds obs vs. SG post $\quad$ ND op 1 ds non-obs: $<0.05$

$98.5 \%$

$10.5 \%$

$\mathrm{ND}$

Table 2 (continued) 
Table 2 (continued)

\begin{tabular}{|c|c|c|c|c|c|c|c|}
\hline Author & Study groups & Biomarker concentrations & Outcomes & AUC on ROC curve & $\mathrm{BCU}$ value & Sensibility & Specificity \\
\hline \multicolumn{8}{|l|}{ ICAM1 ( $n=1 ; 2.6 \%)$} \\
\hline $\begin{array}{l}\text { Taranta-Janusz } \\
\text { et al. (14) }\end{array}$ & $\begin{array}{l}\text { SG: } n=29 ; \text { NSG: } n=23 ; \text { RG: } \\
n=19\end{array}$ & $\begin{array}{l}\text { SG pre op: } 44.36 \mathrm{ng} / \mathrm{mg} \mathrm{Cr} \text { (median); SG post op: } 34.23 \mathrm{ng} / \mathrm{mg} \mathrm{Cr} \text {; } \\
\text { NSG: } 14.98 \mathrm{ng} / \mathrm{mg} \mathrm{Cr} \text {; RG: ND }\end{array}$ & $\begin{array}{l}\text { SG pre op vs. SG post op: NS; SG pre op vs. NSG: }<0.05 \text {; SG post op } \\
\text { vs. NSG: }<0.05 \text {; SG pre op vs. RG: }<0.01\end{array}$ & 0.845 (95\% Cl: $0.728-0.962)$ & $9.486 \mathrm{ng} / \mathrm{mg} \mathrm{Cr}$ & $87.8 \%$ & $70.6 \%$ \\
\hline \multicolumn{8}{|l|}{ HO $1(n=1 ; 2.6 \%)$} \\
\hline Liet al. (31) & $\begin{array}{l}\text { SG: } n=25 ; \text { NSG: } n=25 ; \text { RG: } \\
n=30\end{array}$ & $\begin{array}{l}\text { SG pre op: } 4.23 \mathrm{ng} / \mathrm{mg} \mathrm{Cr} \text { (mean); SG peri op pelvis: } 4.43 \mathrm{ng} / \mathrm{mg} \\
\text { Cr; SG post op } 1 \mathrm{mo:} 1.73 \mathrm{ng} / \mathrm{mg} \mathrm{Cr} \text {; NSG: } 1.04 \mathrm{ng} / \mathrm{mg} \mathrm{Cr} \text {; RG: } \\
0.56 \mathrm{ng} / \mathrm{mg} \mathrm{Cr}\end{array}$ & $\begin{array}{l}\text { SG pre op vs. NSG: }<0.01 \text {; SG pre op vs. RG: }<0.01 \text {; SG peri op pelvis } \\
\text { vs. NSG: }<0.01 \text {; SG peri op pelvis vs. RG: }<0.01 \text { SG post op } 3 \text { mo vs. } \\
\text { NSG: }<0.05 \text {; SG post op } 3 \text { mo vs. RG: }<0.01 \text {; NSG vs. RG: }<0.05\end{array}$ & 0.767 (95\% Cl: $0.624-0.910)$ & $1.92 \mathrm{ng} / \mathrm{mg} \mathrm{Cr}$ & 72.2 & 78.1 \\
\hline \multicolumn{8}{|l|}{ AGT $(n=1 ; 2.6 \%)$} \\
\hline $\begin{array}{l}\text { Taranta-Janusz } \\
\text { et al. (27) }\end{array}$ & $\begin{array}{l}\text { SG: } n=31 \text {; NSG: } n=20 \text {; RG: } \\
n=19\end{array}$ & $\begin{array}{l}\text { SG: } 0.37 \text { ng/mg Cr (median); NSG: } 0.17 \text { ng/mg Cr; RG: } 0.16 \text { ng/mg } \\
\text { Cr }\end{array}$ & $\begin{array}{l}\text { SG vs. NSG: }<0.01 \text {; SG pre op vs. NSG: }<0.05 \text {; SG post op vs. NSG: } \\
<0.05 ; \text { SG vs. RG: <0.01; NSG vs. RG: NS }\end{array}$ & 0.838 (95\% Cl: $0.725-0.951)$ & $0.195 \mathrm{ng} / \mathrm{mg} \mathrm{Cr}$ & $93.3 \%$ & $60.0 \%$ \\
\hline \multicolumn{8}{|l|}{ Emmprin $(n=1 ; 2.6 \%)$} \\
\hline \multicolumn{7}{|l|}{$\operatorname{MMP9}(n=1 ; 2.6 \%)$} & $83 \%$ \\
\hline Tian et al. (19) & SG: $n=15 ;$ NSG: $n=25$ & SG: 33.9 ng/mg Cr (median); NSG: 28.2 ng/mg Cr & SG vs. NSG: $<0.05$ & 0.727 & $27.15 \mathrm{ng} / \mathrm{mg} \mathrm{Cr}$ & $88 \%$ & $67 \%$ \\
\hline \multicolumn{8}{|l|}{$\operatorname{TIMP1}(n=1 ; 2.6 \%)$} \\
\hline Tian et al. (19) & SG: $n=15 ;$ NSG: $n=25$ & SG: 7.4 ng/mg Cr (median); NSG: $5.6 \mathrm{ng} / \mathrm{mg}$ Cr & NS vs. NSG: $<0.05$ & 0.823 & $5.35 \mathrm{ng} / \mathrm{mg} \mathrm{Cr}$ & $80 \%$ & $83 \%$ \\
\hline \multicolumn{8}{|l|}{$\operatorname{MIP1a}(n=1 ; 2.6 \%)$} \\
\hline Madsen et al. (24) & $S G: n=28 ; R G: n=13$ & 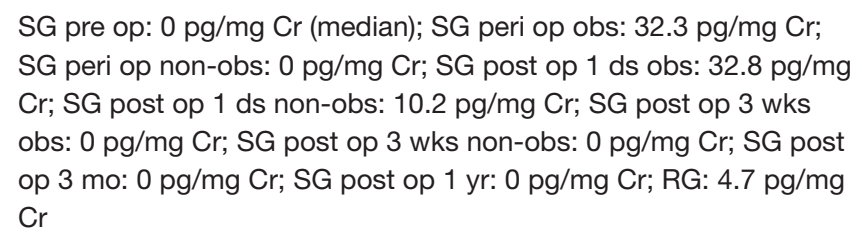 & $\begin{array}{l}\text { SG pre op vs. RG: }<0.05 \text {; SG peri op obs vs. SG peri op non-obs: } \\
<0.05 \text {; SG post op } 1 \text { ds obs vs. RG: <0.05; SG post op } 3 \text { mo vs. RG: } \\
<0.05\end{array}$ & ND & ND & ND & ND \\
\hline \multicolumn{8}{|l|}{ PIIINP (n=1; 2.6\%) } \\
\hline Jianguo et al. (22) & $\begin{array}{l}\text { SG: } n=29 ; \text { NSG: } n=30 ; \text { RG: } \\
n=30\end{array}$ & $\begin{array}{l}\text { SG pre op: } 592.3 \mathrm{pg} / \mathrm{mL} \text { Cr (median); SG } 3 \text { ds post op: } 699 \text { pg/mL } \\
\text { Cr; SG } 3 \text { mo post op: } 307.8 \text { pg/mL Cr; SG } 6 \text { mo post op: } \\
305.1 \text { pg/mL Cr; SG } 1 \text { yr post op: } 322.8 \text { pg/mL Cr; NSG: } \\
264 \text { pg/mL Cr; RG: } 237 \mathrm{pg} / \mathrm{mL} \text { Cr }\end{array}$ & $\begin{array}{l}\text { SG pre op vs. NSG: }<0.01 \text {; SG pre op vs. RG: }<0.01 \text {; SG } 3 \text { ds post op } \\
\text { vs. NSG: }<0.01 ; \text { SG } 3 \text { ds post op vs. RG: }<0.01 ; \text { SG } 3 \text { mo post op vs. } \\
\text { NSG: }<0.01 \text {; SG } 3 \text { mo post op vs. RG: }<0.01 ; \text { SG } 6 \text { mo post op vs. } \\
\text { NSG: }<0.01 ; \text { SG } 6 \text { mo post op vs. RG: }<0.01\end{array}$ & 0.789 (95\% Cl: $0.647-0.930)$ & $334.6 \mathrm{pg} / \mathrm{mL} \mathrm{Cr}$ & $88.2 \%$ & $69 \%$ \\
\hline \multicolumn{8}{|c|}{ Exoglycosidases $(n=1 ; 2.6 \%)$} \\
\hline $\begin{array}{l}\text { Taranta-Janusz } \\
\text { et al. (16) }\end{array}$ & $\begin{array}{l}\text { SG: } n=16 ; \text { NSG: } n=16 ; R G: \\
n=42\end{array}$ & 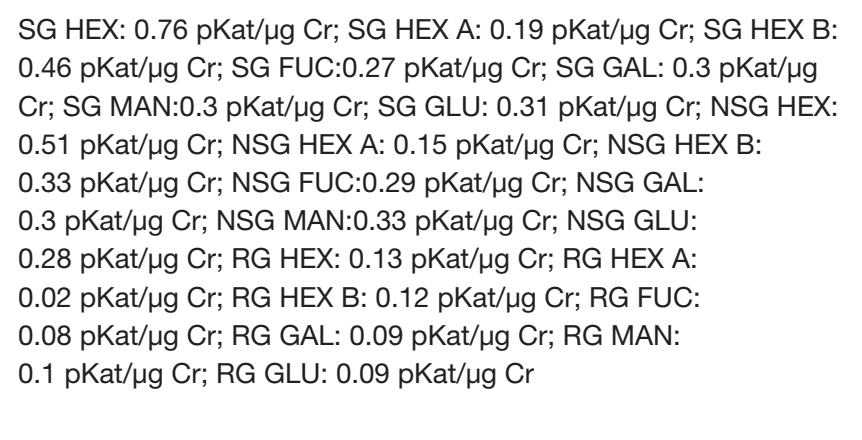 & 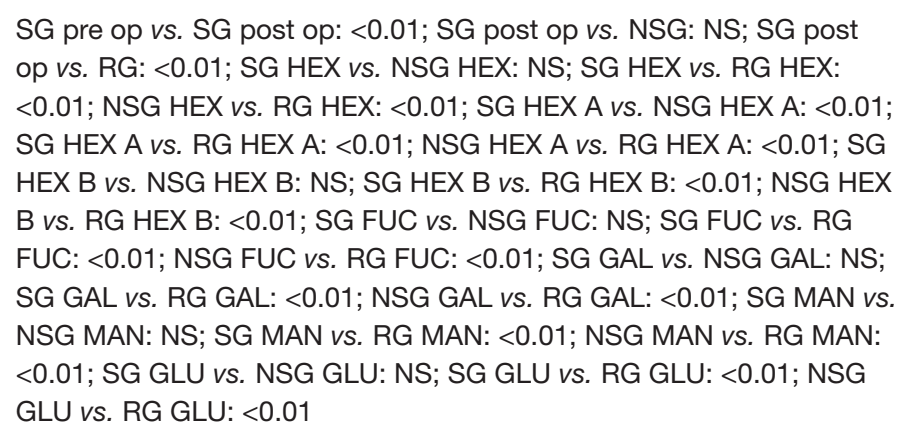 & $\begin{array}{l}\text { HEX: } 0.869 ; \text { HEX A: 0.846; HEX B: } \\
\text { 0.858; FUC: } 0.837 ; \text { GAL: } 0.798 ; \\
\text { MAN: } 0.821 ; \text { GLU: } 0.814\end{array}$ & $\begin{array}{l}\text { HEX: } 0.309 \mathrm{pKat} / \mathrm{\mu g} \mathrm{Cr} ; \\
\text { HEX A: } 0.097 \mathrm{pKat} / \mathrm{\mu g} \text { Cr; } \\
\text { HEX B: } 0.306 \mathrm{pKat} / \mathrm{\mu g} \\
\text { Cr; FUC: } 0.2 \mathrm{pKat} / \mathrm{\mu g} \mathrm{Cr} \text {; } \\
\text { GAL: } 0.156 \mathrm{pKat} / \mu \mathrm{g} \mathrm{Cr} ; \\
\text { MAN: } 0.204 \mathrm{pKat} / \mathrm{\mu g} \mathrm{Cr} ; \\
\text { GLU: } 0.171 \mathrm{pKat} / \mathrm{\mu g} \mathrm{Cr}\end{array}$ & $\begin{array}{l}\text { HEX: } 93.8 \% \text {; } \\
\text { HEX A: } 84.4 \% \text {; } \\
\text { HEX B: } 68.8 \% \text {; } \\
\text { FUC: } 81.3 \% \text {; } \\
\text { GAL: } 87.5 \% ; \\
\text { MAN: } 87.5 \% ; \\
\text { GLU: } 84.4 \%\end{array}$ & $\begin{array}{l}\text { HEX: } 76.2 \% \text {; } \\
\text { HEX A: } 78.6 \% \text {; } \\
\text { HEX B: } 90.5 \% \text {; } \\
\text { FUC: } 81.0 \% \text {; } \\
\text { GAL: } 71.4 \% ; \\
\text { MAN: } 76.2 \% ; \\
\text { GLU: } 73.8 \%\end{array}$ \\
\hline \multicolumn{8}{|l|}{$\mathrm{CD} 10(\mathrm{n}=1 ; 2.6 \%)$} \\
\hline Gerber et al. (13) & $S G: n=12 ; R G: n=12$ & SG: $8.94 / \mathrm{Cr}$ (mean); RG: $2.98 / \mathrm{Cr}$ & SG vs. RG: $<0.01$ & ND & ND & ND & ND \\
\hline
\end{tabular}

Table 2 (continued) 


\begin{tabular}{|c|c|c|c|c|c|c|c|}
\hline Author & Study groups & Biomarker concentrations & Outcomes & AUC on ROC curve & $\mathrm{BCU}$ value & Sensibility & Specificity \\
\hline \multicolumn{8}{|l|}{$\mathrm{CD} 13(\mathrm{n}=1 ; 2.6 \%)$} \\
\hline Gerber et al. (13) & $S G: n=12 ; R G: n=12$ & SG: 10.84/Cr (mean); RG: 1.15/Cr & SG vs. RG: $<0.05$ & ND & ND & ND & ND \\
\hline \multicolumn{8}{|l|}{$\operatorname{CD} 26(n=1 ; 2.6 \%)$} \\
\hline Gerber et al. (13) & SG: $n=12 ; R G: n=12$ & SG: $978.57 / \mathrm{Cr}$ (mean); RG: 90.56/Cr & SG vs. RG: $<0.01$ & ND & ND & ND & ND \\
\hline \multicolumn{8}{|l|}{$\alpha$-GST $(n=1 ; 2.6 \%)$} \\
\hline Bieniaś et al. $(7)^{\star}$ & $\begin{array}{l}\text { Group A: } n=25 ; \text { group B: } \\
n=11 ; \text { group C: } n=9 ; R G: \\
n=21 ; \text { ON: } n=28 ; \text { NON: } n=17\end{array}$ & $\begin{array}{l}\text { Group A: } 4.51 \mathrm{ng} / \mathrm{mg} \mathrm{Cr} \text { (median); group B: } 6.17 \mathrm{ng} / \mathrm{mg} \mathrm{Cr} \text {; } \\
\text { group C: } 4.73 \mathrm{ng} / \mathrm{mg} \text { Cr; RG: } 1.11 \mathrm{ng} / \mathrm{mg} \mathrm{Cr}\end{array}$ & $\begin{array}{l}\text { Group A vs. control group: } 0.0001 \text {; group B vs. control group: } 0.008 ; \\
\text { group C vs. control group: } 0.008 \text {; ON vs. NON: } 0.03\end{array}$ & $\begin{array}{l}\text { ON vs. RG: 0.902; ON vs. NON: } \\
0.750\end{array}$ & $\begin{array}{l}\text { ON vs. RG: } 0.046 \mathrm{ng} / \mathrm{mg} \\
\text { Cr; ON vs. NON: } \\
0.098 \mathrm{ng} / \mathrm{mg} \mathrm{Cr}\end{array}$ & $\begin{array}{l}\text { ON vs. RG: } \\
81.8 \% \text {; ON vs. } \\
\text { NON: } 84.6 \%\end{array}$ & $\begin{array}{l}\text { ON vs. RG: } \\
84.6 \% \text {; ON vs. } \\
\text { NON: } 69.2 \%\end{array}$ \\
\hline Bieniaś et al. (7)* & $\begin{array}{l}\text { Group A: } n=25 ; \text { group B: } \\
\mathrm{n}=11 ; \text { group } \mathrm{C}: \mathrm{n}=9 ; \mathrm{RG}: \\
\mathrm{n}=21 ; \mathrm{ON}: \mathrm{n}=28 ; \mathrm{NON}: \mathrm{n}=17\end{array}$ & $\begin{array}{l}\text { Group A: } 30.4 \mathrm{ng} / \mathrm{mg} \mathrm{Cr} \text { (median); group B: } 17.9 \mathrm{ng} / \mathrm{mg} \mathrm{Cr} \text {; } \\
\text { group C: } 16.3 \mathrm{ng} / \mathrm{mg} \mathrm{Cr} ; \mathrm{RG}: 14.6 \mathrm{ng} / \mathrm{mg} \mathrm{Cr}\end{array}$ & $\begin{array}{l}\text { Group A vs. control group: } 0.03 \text {; group B vs. control group: ns; } \\
\text { group C vs. control group: NS; ON vs. NON: NS }\end{array}$ & ON vs. RG: 0.3; ON vs. NON: 0.574 & $\begin{array}{l}\text { ON vs. RG: } 0.082 \text { ng/mg } \\
\text { Cr; ON vs. NON: } \\
0.103 \text { ng/mg Cr }\end{array}$ & $\begin{array}{l}\text { ON vs. RG: } \\
\text { 92.3\%; ON vs. } \\
\text { NON: } 92.3 \%\end{array}$ & $\begin{array}{l}\text { ON vs. RG: } \\
<1 \% \text {; ON vs. } \\
\text { NON: } 7.7 \%\end{array}$ \\
\hline \multicolumn{8}{|l|}{ IL6 ( $(n=1 ; 2.6 \%)$} \\
\hline Yu et al. (5) & UPJO: $n=17 ;$ RG: $n=17$ & ND & $\begin{array}{l}\text { UPJO vs. RG: }<0.0073 \text {; bilateral UPJO vs. unilateral UPJO: }<0.007 \text {; } \\
\text { bilateral UPJO vs. RG: }<0.03\end{array}$ & 0.78 & ND & ND & ND \\
\hline \multicolumn{8}{|l|}{$B D 1(n=1 ; 2.6 \%)$} \\
\hline Gupta et al. (8) & $S G: n=30 ; R G: n=15$ & SG: $109.10 \mathrm{ng} / \mathrm{mg}$ Cr (median); RG: 206.40 ng/mg Cr & SG vs. RG: 0.015 & 0.73 & $152.8 \mathrm{ng} / \mathrm{mg} \mathrm{Cr}$ & $71.4 \%$ & $80.8 \%$ \\
\hline \multicolumn{8}{|c|}{ Cathelicidin (LL37) $(n=1 ; 2.6 \%)$} \\
\hline Gupta et al. (8) & $S G: n=30 ; R G: n=15$ & SG: 0.03 ng/mg Cr (median); RG: 0.07 ng/mg Cr & SG vs. RG: 0.0461 & 0.68 & $0.033 \mathrm{ng} / \mathrm{mg} \mathrm{Cr}$ & $53.3 \%$ & $86.7 \%$ \\
\hline \multicolumn{8}{|l|}{ HD5 (n=1; 2.6\%) } \\
\hline Gupta et al. (8) & $S G: n=30 ; R G: n=15$ & SG: 0.24 ng/mg Cr (median); RG: 0.36 ng/mg Cr & SG vs. RG: NS & ND & ND & ND & ND \\
\hline \multicolumn{8}{|l|}{$\operatorname{Trx}(n=1 ; 2.6 \%)$} \\
\hline Xuet al. (12) & SG: $n=156 ; R G: n=80$ & ND & SG vs. RG: $<0.001$ & ND & $\begin{array}{l}\text { SRF }<39.2 \%: \\
21.3 \mathrm{ng} / \mathrm{mL} ; \mathrm{APD} \\
>30 \mathrm{~mm}: 22.1 \mathrm{ng} / \mathrm{mL} ; \\
\text { grade IV HN: } 27.1 \mathrm{ng} / \mathrm{mL}\end{array}$ & $\begin{array}{l}85.9 \% ; 78.2 \% \\
66.2 \%\end{array}$ & $\begin{array}{l}64.1 \% ; 66.7 \% \\
75.0 \%\end{array}$ \\
\hline \multicolumn{8}{|c|}{ Semaphorin $3 A(n=1 ; 2.6 \%)$} \\
\hline Liet al. (9) & $\begin{array}{l}\text { SG: } n=42 ; N S G: n=42 ; R G: \\
n=44\end{array}$ & $\begin{array}{l}\text { SG pre op: } 256.1 \text { pg/mg Cr (median); SG pelvis: } 391.5 \text { pg/mg Cr; } \\
\text { SG } 1 \text { mo post op: } 166.6 \text { pg/mg Cr; NSG: } 82.1 \text { pg/mg Cr; RG: } 48.1 \\
\text { pg/mg Cr }\end{array}$ & $\begin{array}{l}\text { SG pre op vs. NSG: }<0.01 \text {; SG pelvis vs. NSG: }<0.01 \text {; SG pre op vs. } \\
\text { RG: }<0.01 \text {; SG pelvis vs. RG: <0.01; SG } 1 \text { mo post op vs. NSG: }<0.05 \text {; } \\
\text { SG } 1 \text { mo post op vs. RG: }<0.01\end{array}$ & 0.825 (95\% Cl: 0.691-0.960) & $186.65 \mathrm{pg} / \mathrm{mg} \mathrm{Cr}$ & $89.5 \%$ & $80 \%$ \\
\hline \multicolumn{8}{|l|}{ L-FABP $(n=1 ; 2.6 \%)$} \\
\hline Noyan et al. (17) & $\begin{array}{l}\text { SG: } n=26 \text {; NSG: } n=36 \text {; RG: } \\
n=20\end{array}$ & $\begin{array}{l}\text { SG: } 1.2 \mathrm{ng} / \mathrm{mg} \mathrm{Cr}[0-15] \text { (median); NSG: } 1.5 \mathrm{ng} / \mathrm{mg} \mathrm{Cr} \text { [0-11]; RG: } \\
0.8 \mathrm{ng} / \mathrm{mg} \mathrm{Cr}[0-7]\end{array}$ & SG vs. NSG: NS; SG vs. RG: NS & ND & ND & ND & ND \\
\hline \multicolumn{8}{|l|}{ Netrin $1(n=1 ; 2.6 \%)$} \\
\hline Liet al. (9) & $\begin{array}{l}\text { SG: } n=42 ; N S G: n=42 ; R G: \\
n=44\end{array}$ & $\begin{array}{l}\text { SG pre op: } 736.9 \text { pg/mg Cr (median); SG pelvis: } 822.7 \text { pg/mg Cr; } \\
\text { SG } 1 \mathrm{mo} \mathrm{post} \mathrm{op:} 604.6 \mathrm{pg} / \mathrm{mg} \text { Cr; NSG: } 488.5 \text { pg/mg Cr; RG: } \\
287.7 \mathrm{pg} / \mathrm{mg} \mathrm{Cr}\end{array}$ & $\begin{array}{l}\text { SG pre op vs. NSG: }<0.01 \text {; } S \text { pelvis vs. NSG: }<0.01 ; \text { SG pre op vs. } \\
\text { RG: }<0.01 ; \text { SG pelvis vs. RG: }<0.01 ; \text { SG } 1 \text { mo post op vs. NSG: }<0.01 \text {; } \\
\text { SG } 1 \text { mo post op vs. RG: }<0.01\end{array}$ & 0.745 (95\% Cl: $0.577-0.914)$ & $642.95 \mathrm{pg} / \mathrm{mg} \mathrm{Cr}$ & $73.7 \%$ & $80 \%$ \\
\hline
\end{tabular}

Table 2 (continued) 
Table 2 (continued) Study groups Biomarker concentrations Outcomes AUC on ROC curve

BCU value Sensibility Specificity

Shirazi et al. (11) SG: $n=31 ; R G: n=33$

SG pre op: $22.38 \mathrm{ng} / \mathrm{mg} \mathrm{Cr}$ (mean); SG 3 mo post op: $13.90 \mathrm{ng} / \mathrm{mg}$ Cr. SG 6 mo post op: $8.34 \mathrm{ng} / \mathrm{mg} \mathrm{Cr}$

Gep vs. SG 3 mo post op vs. 6 mo post op: <0.01; SG pre op vs. ND RG: <0 001. SG 3 mo post op vs. RG: NS

$\operatorname{TNF} \alpha(n=1 ; 2.6 \%)$

Shirazi et al. (11) SG: $n=31 ; R G: n=33$

SG pre op: 80.00 pg/mg Cr (mean); SG 3 mo post op: 32.89 pg/mg Cr; SG 6 mo post op: $13.34 \mathrm{pg} / \mathrm{mg} \mathrm{Cr}$

RG: $<0.001$. SG 3 mo post op vs. 6 mo po

ND

RG. $<0.001$; SG 3 mo post op vs. RG: NS

SFAS/APO-1 ( $n=1 ; 2.6 \%)$

Gawtowska- $\quad$ SG: $n=45$ (young children: adolescents: $n=18): n=20$;

older children: $n=5$;

SG pelvic urine young children $3.19 \mathrm{ng} / \mathrm{mg} \mathrm{Cr}$ (mean); SG pelvic urine older children $3.17 \mathrm{ng} / \mathrm{mg} \mathrm{Cr} ;$ SG pelvic urine adolescents

(mean); SG bladder urine older children $3.24 \mathrm{ng} / \mathrm{mg} \mathrm{Cr}$; SG bladder $\quad$ adolescents: $<0.05$; SG bladder urine young children vs. RG bladder

SG pelvic urine young children $v$ s. RG bladder urine young children:
$<0.05 ; S G$ pelvic urine older children vs. RG bladder urine older $<0.05$; SG pelvic urine older children vs. RG bladder urine older

$1.07 \mathrm{ng} / \mathrm{mg} \mathrm{Cr}$ (mean): RG bladder urine older children $0.98 \mathrm{ng} / \mathrm{mg}$

$\mathrm{Cr}$ : RG bladder urine adolescents $0.33 \mathrm{ng} / \mathrm{mg} \mathrm{Cr}$; SG bladder urine

young children $12 \mathrm{mo}$ post op $1.45 \mathrm{ng} / \mathrm{mg} \mathrm{Cr}$ (mean); SG bladder

e young children: $<0.05$; $S G$ bladder urine older children $v s . \mathrm{RG}$

RG bladder urine adolescents: 00.05 : SG bladder urine young children

vs. SG bladder urine young children 12 mo post op $<0.05$; SG bladder

urine older children vs. SG bladder urine older children 12 mo post op adolescents children $12 \mathrm{mo}$ post op $0.98 \mathrm{ng} / \mathrm{mg} \mathrm{C}$

min outcomes investigated; value of the AUC on ROC curve; BCO value of urinary biomarker concentration; sensibility; specificity. * The patients were divided into three

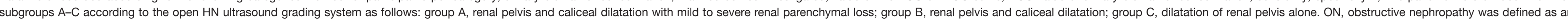

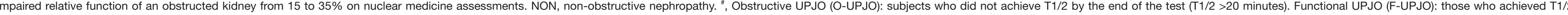

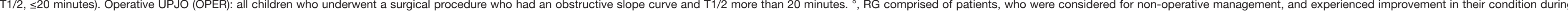

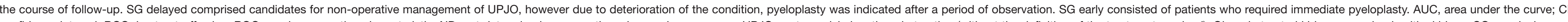

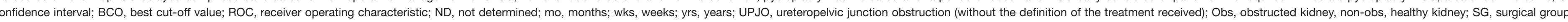
NSG, non-surical gro, Bes, 


\section{MCP1}

Several studies found that MCP1 levels were significantly increased in preoperative samples compared to healthy controls $(10,15,24,29,42)$. Some authors $(10,15,20,29)$ exhibited that MCP1 concentrations were greater in the surgical PUJO group compared to patients with mild non-obstructive hydronephrosis. Additionally, in the SG, the difference between the pre- and post-operative concentrations became significant lower between 2 and 4 months after surgery according to Grandaliano et al. (42).

\section{$E G F$}

Preoperative concentrations of urinary EGF were significantly increased compared to healthy controls according to Madsen et al. (24) and compared to children with dilated non-obstructed kidneys according to Mohammadjafari et al. (21). Conversely, data reported from Grandaliano et al. (42) showed significantly lower urinary EGF concentrations in the SG compared to healthy children and they were also significantly lower compared to the observational group according to Li et al. (28).

However, in the series reported by Taha et al. (39) no difference was identified between surgical patients and healthy children. Madsen et al. (24), Taha et al. (39) and Grandaliano et al. (42) considered the evolution of EGF concentrations after pyeloplasty: postoperative values decreased significantly between 2 and 4 months after surgery according to Grandaliano et al. (42), while no difference was reported by Madsen et al. (24) and by Taha et al. (39) 1 year after surgery.

\section{KIM1}

Preoperative values of KIM1 were reported significantly augmented compared to healthy controls by Karakus et al. (15) by Wasilewska et al. (32) and compared to conservative cases by Mohammadjafari et al. (21) and by Wasilewska et al. (32). Preoperative values of KIM1 were reported not significantly different compared to controls by Noyan et al. (17) Gerber et al. (13) and compared to conservative cases by Karakus et al. (15) and Noyan et al. (17).

\section{CCL5/RANTES}

Three studies analysed CCL5/RANTES as a promising biomarker of PUJO. According to Madsen et al. (24) and to
Taranta-Janusz et al. (29) there was a significant difference between the CCL/RANTES concentration obtained from the pelvic urine of surgical PUJO patients and those obtained from the bladder of healthy children. This difference became insignificant postoperatively according to Madsen et al. (24) but not to Taranta-Janusz et al. (29).

\section{CA19.9}

Three studies $(6,18,34)$ reported that preoperative CA19.9 values were significantly higher in the SG compared to the healthy RG. Atar et al. (18) also demonstrated that surgical PUJO patients have significantly higher preoperative values compared to dilated non-obstructive patients. Three months after surgery, the voided urine CA19.9 levels decreased significantly according to Atar et al. (18) and Kajbafzadeh et al. (34).

\section{$N A G$}

Two studies $(36,37)$ stated that preoperative levels of NAG were significantly increased compared to the conservative cases. In addition, Taha et al. (37) demonstrated that younger children had significantly higher activities of voided urinary NAG compared to older ones at diagnosis.

\section{Other markers}

The other biomarkers were evaluated in two studies or less with different results and degrees of predicting success (See Table 2).

\section{Discussion}

Obstructive nephropathy represents the major cause of renal insufficiency in children and, among them, PUJO constitutes the most frequent condition (1). Currently, according to the European Association of Urology Guidelines, symptomatic obstruction, impaired DRF (less than $40 \%$ ), decrease of more than $10 \%$ of renal function in subsequent investigations, poor drainage function after the administration of diuretic, increasing anteroposterior pelvic diameter (APD) on US, and grade III and IV hydronephrosis represent indications for surgery (43). However, in many cases, patients present border-line results that make difficult to predict whether the affected kidney is a risk of damage and, in some, the indication for surgery comes only after several radiological investigations, 
often when the evidence that a renal damage has already established.

A biomarker has been defined as a characteristic that is objectively measured and evaluated as an indicator of normal biological processes, pathogenic processes, or pharmacologic responses to a therapeutic intervention (44). Animal model systems have provided new insights into the cellular response of the developing kidney to urinary tract obstruction, helping in identify molecules involved in the pathogenic response to kidney damage, mediators of interstitial inflammation, tubular apoptosis and fibrosis. They became targets of proteomic studies aiming to identify early predictors of renal sufferance that could be used for clinical decision-making, assisting in earlier and more reliable determination of patients at risk to develop renal damage, guiding more timely therapeutic decisions and monitoring the treatment efficacy.

In addition to their diagnostic relevance, they could also prove to be extremely useful in the designing molecular therapies to prevent or reverse the renal structural and functional consequences of obstructive nephropathy. We aimed to perform a systematic review of the published literature of urinary biomarkers used as a tool for diagnosis and prognosis of children with PUJO.

\section{Analysis of the most used markers}

\section{NGAL}

NGAL is a non-organ specific protein of the lipocalin family which is secreted by different tissues and is reabsorbed via epithelia endocytosis in the proximal renal tubules. Its expression is also induced in the thick ascending limb of Henle's loop and in the collecting ducts in response to renal tubular injury. Thus, the combination of increased gene expression in the distal nephron and altered reabsorption in the proximal tubule make it one of the first indicators of renal damage in case of obstruction. Case-control prospective studies in children with severe hydronephrosis secondary to PUJO suggested that urine NGAL/creatinine ratio from bladder was significantly higher in affected patients compared to normal subjects $(5,7,8,15,17,26,32)$.

Although, Madsen et al. (30) didn't find any significant differences between bladder urine NGAL levels in PUJO patients and in controls, renal pelvis NGAL levels in obstructed kidneys were significantly higher than those in the bladder and the levels measured in the voided urine of healthy children. These data were confirmed by Cost et al. (26). NGAL levels tend to decrease at 3 to 6 months after surgery $(15,30,32)$.

Finally, ROC curve was used to determine the BCO point of urinary NGAL levels in predicting PUJO patients and AUC and the corresponding 95\% CI were calculated. The best performance was reported by Madsen et al. (30) who found at a cut off value of $20.57 \mathrm{ng} / \mathrm{mg} \mathrm{Cr}$, an AUC of 0.923 [95\% confidence interval (CI), 0.837-1.000] which yielded $82 \%$ sensitivity and $100 \%$ specificity.

\section{TGFß1}

TGF $\beta 1$ is a proinflammatory, proapoptotic, profibrotic cytokine and fibroblast chemoattractant which plays a major role in renal fibrosis, also via epithelial-mesenchymal transition. Up-regulation of TGF $\beta 1$ synthesis in the kidney is associated with accumulation of collagen and scarring, leading to the development of advanced chronic renal disease.

Experimental models documented that kidney responds to obstruction inducing a cascade of molecular events and histological changes starting from the up-regulation of the renin-angiotensin system, which increase the expression of tissue TGF $\beta 1$. The deduction is that its level could be used as a non-invasive tool to evaluate the progression of renal disease and to monitor the efficacy of surgery. TGF $\beta 1$ levels are remarkably elevated in surgical PUJO patients in confront to healthy children $(23,35,39)$. Moreover, postoperative mean TGF $\beta 1$ concentration was significantly lower than preoperative TGF $\beta 1$, according to Sager et al. (35) and Taha et al. (39).

\section{MCP1}

MCP1 is a chemokine that promotes monocyte chemotaxis. In case of obstruction, its expression at the tubular level seems to be strictly associated with the recruitment of these inflammatory cells within the interstitial space. Thus, MCP1 urine excretion could be related to the extent of monocyte infiltration and the consequent progression of interstitial renal fibrosis.

All studies agreed in considering MCP1 levels highly related to the extent of tubular atrophy and interstitial fibrosis and demonstrated that its urinary concentration could successfully discriminate not only between children who require an intervention and healthy controls but also within the hydronephrosis group. In fact, various authors found that MCP1 urinary levels are significantly increased in surgical patients compared to healthy controls $(5,10,15,24,29,42)$ and compared to children managed conservatively $(10,15,20,29)$. 
MCP1 could be used for long term follow-up in the postoperative period since its levels decreased and became similar to the healthy group between 2 and 4 months after surgery $(15,24,42)$. Analysing the ROC curves, MCP1 maintained also a good diagnostic profile and therefore may be used to distinguish surgical PUJO patients.

\section{EGF}

EGF is a polypeptide growth factor which plays a fundamental role in normal tubulogenesis. It is synthesized by the ascending portion of Henle's loop and by the distal convoluted tubule and displays fundamental effects on intact glomeruli, proximal tubules and collecting ducts. It was demonstrated that it has a central role in modulating tubular cell growth and tissue response in kidneys with tubule interstitial injury. However, the evaluation of urinary EGF concentration in PUJO children is still not fully understood and the studies that have investigated its urinary expression described contradictory results. This could be due to the fact that different methods were used in the studies: two studies considered also patients with bilateral disease, two reported also the concentration of the biomarker collected from the pelvic urine and two studies compared the urinary level of children with surgical PUJO with children with dilated but non-obstructed kidneys. Particularly, EGF urinary levels in patients undergoing surgery were significantly higher compared to those treated conservatively in the study of Mohammadjafari et al. (21) but they were significantly lower in the study of $\mathrm{Li}$ et al. (28). Although Grandaliano et al. (42) claimed that the preoperative concentrations of urinary EGF were significantly reduced in PUJO patients in comparison to their healthy controls, Madsen et al. (24) found that they were more elevated. To further confuse the situation, Taha et al. (39) reported that there was no significant difference of EGF values between surgical patients and controls and moreover there was no significant difference between preoperative and postoperative values even 1 year after surgery. In conclusion, the role of EGF as a urinary marker of PUJO seems, at least, debatable.

\section{KIM1}

KIM1 is a member of the type I transmembrane glycoprotein. Since it is undetectable in healthy children but is strongly expressed and released by damaged proximal tubular epithelial cells until complete recovery, it could be utilised as an early and sensitive biomarker for kidney injury in the setting of obstructive nephropathy.

In this scenario, several clinical studies have shown that urinary KIM1 is higher in patients with PUJO compared to control groups (15) and to children with mild hydronephrosis $(21,32)$. However, other studies failed in identifying differences between PUJO patients and healthy controls $(13,17)$ or hydronephrotic patients $(15,17)$. KIM1 levels have a tendency to decrease and normalize by 3 months after surgery $(15,32)$. ROC analyses were performed to define the diagnostic profile of KIM1 in identifying children with an obstructed kidney condition with quite good results. The best AUC (0.89) was reported by Karakus et al. (15) with a cut-off value of $0.687 \mathrm{ng} / \mathrm{mg}$ Cr, which gave a sensibility of $92.3 \%$ and a specificity of $83.3 \%$. It is, furthermore, possible to conclude that KIM1 urinary levels are closely related to the severity of renal damage, since a negative correlation was found with DRF in radionuclide scans (32). However, Gerber et al. (13) didn't identify any significant linear correlations. This could have been consequence of the small sample size of their study or the presence of outliers in their small datasets, making any potential correlations difficult to demonstrate. No significant correlation was found with initial anterior posterior (AP) diameter (length) of the pelvis.

\section{Critical review}

Recently much has been learned about the pathophysiology of obstructive nephropathy and many novel biomarkers have been investigated for diagnostic and prognostic purposes. A number of endogenous molecules have been identified with different degrees of success, often with contradictory results. This wide range of outcomes could be due to a variety of reasons. First of all, the populations considered in the different articles were quite heterogeneous. Secondly, the heterogeneity of the populations considered and the different criteria adopted to classify patients made virtually impossible to compare the results. In addition to this, also the study designs were extremely wide-ranging. Another important bias was that these studies were held utilizing a wide range of patient ages. Elder children could therefore have suffered from ureterovascular hydronephrosis due to aberrant renal vessels. In these cases, decrements in renal function may occur discontinuously, inevitably interfering only episodically with urinary biomarker concentrations, which decrease during the diuretic phase of the renal impairment. In addition, it is also possible that 
the progression of the histopathological tubulointerstitial changes is extremely different between the age groups. Finally, the duration of follow-up was quite short especially in the more recent studies. All these shortcomings could be overcome by large prospective clinical trials with appropriate and strictly design protocols.

Urinary biomarkers have been extensively used as a promising tool for non-invasive assessment of PUJO in children. They could be helpful not only in the diagnosis of congenital obstructive uropathies but also in the differentiation between dilated but non-obstructed kidneys that could be managed conservatively.

Some studies also demonstrated that the urinary biomarkers could be useful in the evaluation of the surgical treatment success. Nevertheless, the existing literature is still lacking of solid and definitive studies. Large multicentre and carefully designed prospective studies are needed to evaluate the clinical usefulness of urinary biomarkers in the diagnosis and follow-up of children with obstructive nephropathy.

\section{Acknowledgments}

Funding: None.

\section{Footnote}

Conflicts of Interest: All authors have completed the ICMJE uniform disclosure form (available at http://dx.doi. org/10.21037/tau.2020.01.01). NRD and CT report other from SpOtOn Clinical Diagnostics Ltd, NRD and CT have a patent Stable isotope albumin peptide internal standard issued. The other authors have no conflicts of interest to declare.

Ethical Statement: The authors are accountable for all aspects of the work in ensuring that questions related to the accuracy or integrity of any part of the work are appropriately investigated and resolved.

Open Access Statement: This is an Open Access article distributed in accordance with the Creative Commons Attribution-NonCommercial-NoDerivs 4.0 International License (CC BY-NC-ND 4.0), which permits the noncommercial replication and distribution of the article with the strict proviso that no changes or edits are made and the original work is properly cited (including links to both the formal publication through the relevant DOI and the license). See: https://creativecommons.org/licenses/by-nc$\mathrm{nd} / 4.0 /$.

\section{References}

1. Harambat J, van Stralen KJ, Kim JJ, et al. Epidemiology of chronic kidney disease in children. Pediatr Nephrol 2012 Mar;27:363-73.

2. Nguyen HT, Herndon CD, Cooper C, et al. The Society for Fetal Urology consensus statement on the evaluation and management of antenatal hydronephrosis. J Pediatr Urol 2010;6:212-31.

3. Heinlen JE, Manatt CS, Bright BC, et al. Operative versus nonoperative management of ureteropelvic junction obstruction in children. Urology 2009;73:521-5; discussion 525 .

4. Chevalier RL. Obstructive nephropathy: towards biomarker discovery and gene therapy. Nat Clin Pract Nephrol 2006;2:157-68. Review.

5. Yu L, Zhou L, Li Q, et al. Elevated urinary lipocalin-2, interleukin-6 and monocyte chemoattractant protein-1 levels in children with congenital ureteropelvic junction obstruction. J Pediatr Urol 2019;15:44.e1-7.

6. Nabavizadeh B, Khorramirouz R, Amini E, et al. Value of urinary carbohydrate antigen 19-9 to predict failure of conservative management in children with ureteropelvic junction obstruction. J Pediatr Surg 2019;54:1650-3.

7. Bieniaś B, Sikora P. Potential novel biomarkers of obstructive nephropathy in children with hydronephrosis. Dis Markers 2018;2018:1015726.

8. Gupta S, Jackson AR, DaJusta DG, et al. Urinary antimicrobial peptides: potential novel biomarkers of obstructive uropathy. J Pediatr Urol 2018;14:238.e1-6.

9. Li X, Liu X, Li J, et al. Semaphorin-3A and Netrin-1 predict the development of kidney injury in children with congenital hydronephrosis. Scand J Clin Lab Invest 2018;78:55-61.

10. Sadeghi-Bojd S, Alijani E, Mahdavian MS. Mean urinary cytokine MCP-1 in children with urinary tract obstruction and healthy children. Nephro-Urol Mon 2018;10:e63607.

11. Shirazi M, Eslahi A, Sharifi V, et al. Evaluation of caspase 3 enzyme and TNF-alpha as biomarkers in ureteropelvic junction obstruction in children- a preliminary report. Pak J Med Sci 2017;33:315-9.

12. Xu ZM, Li MJ, Tao C. Serum and urinary thioredoxin concentrations are associated with severity of children hydronephrosis. Clin Chim Acta 2017;466:127-32.

13. Gerber C, Harel M, Lynch ML, et al. Proximal tubule 
proteins are significantly elevated in bladder urine of patients with ureteropelvic junction obstruction and may represent novel biomarkers: a pilot study. J Pediatr Urol 2016;12:120.e1-7.

14. Taranta-Janusz K, Wasilewska A, Roszkowska R, et al. Is urine intercellular adhesion molecule- 1 a marker of renal disorder in children with ureteropelvic junction obstruction? Biomarkers 2016;21:123-8.

15. Karakus S, Oktar T, Kucukgergin C, et al. Urinary IP10, MCP-1, NGAL, cystatin-C, and KIM-1 levels in prenatally diagnosed unilateral hydronephrosis: the search for an ideal biomarker. Urology 2016;87:185-92.

16. Taranta-Janusz K, Zalewska-Szajda B, Chojnowska S, et al. Urine exoglycosidases are potential markers of renal tubular injury in children with ureteropelvic junction obstruction. Acta Paediatr 2015;104:e518-23.

17. Noyan A, Parmaksiz G, Dursun H, et al. Urinary NGAL, KIM-1 and L-FABP concentrations in antenatal hydronephrosis. J Pediatr Urol 2015;11:249.e1-6.

18. Atar A, Oktar T, Kucukgergin C, et al. The roles of serum and urinary carbohydrate antigen 19-9 in the management of patients with antenatal hydronephrosis. J Pediatr Urol 2015;11:133.e1-5.

19. Tian F, Gu C, Zhao Z, et al. Urinary Emmprin, matrix metalloproteinase 9 and tissue inhibitor of metalloproteinase 1 as potential biomarkers in children with ureteropelvic junction narrowing on conservative treatment. Nephrology (Carlton) 2015;20:194-200.

20. Mohammadjafari H, Rafiei A, Mousavi SA, et al. Role of urinary levels of endothelin-1, monocyte chemotactic peptide-1, and $\mathrm{N}$-acetyl glucosaminidase in predicting the severity of obstruction in hydronephrotic neonates. Korean J Urol 2014;55:670-6.

21. Mohammadjafari H, Rafiei A, Kosaryan M, et al. Determination of the severity of ureteropelvic junction obstruction using urinary epidermal growth factor and kidney injury molecule 1 levels. Biomark Med 2014;8:1199-206.

22. Jianguo $W$, Zhenzhen L, Xianghua L, et al. Serum and urinary procollagen III aminoterminal propeptide as a biomarker of obstructive nephropathy in children. Clin Chim Acta 2014;434:29-33.

23. Merrikhi A, Bahraminia E. Association of urinary transforming growth factor- $\beta 1$ with the ureteropelvic junction obstruction. Adv Biomed Res 2014;3:123.

24. Madsen MG, Nørregaard R, Palmfeldt J, et al. Epidermal growth factor and monocyte chemotactic peptide-1: potential biomarkers of urinary tract obstruction in children with hydronephrosis. J Pediatr Urol 2013;9:838-45.

25. Gawłowska-Marciniak A, Niedzielski JK. Evaluation of TGF- $\beta 1$, CCL5/RANTES and sFas/Apo-1 urine concentration in children with ureteropelvic junction obstruction. Arch Med Sci 2013;9:888-94.

26. Cost NG, Noh PH, Devarajan P, et al. Urinary NGAL levels correlate with differential renal function in patients with ureteropelvic junction obstruction undergoing pyeloplasty. J Urol 2013;190:1462-7.

27. Taranta-Janusz K, Wasilewska A, Dębek W, et al. Urinary angiotensinogen as a novel marker of obstructive nephropathy in children. Acta Paediatr 2013;102:e429-33.

28. Li Z, Zhao Z, Liu X, et al. Prediction of the outcome of antenatal hydronephrosis: significance of urinary EGF. Pediatr Nephrol 2012;27:2251-9.

29. Taranta-Janusz K, Wasilewska A, Dębek W, et al. Urinary cytokine profiles in unilateral congenital hydronephrosis. Pediatr Nephrol 2012;27:2107-13.

30. Madsen MG, Nørregaard R, Palmfeldt J, et al. Urinary NGAL, cystatin C, $\beta 2$-microglobulin, and osteopontin significance in hydronephrotic children. Pediatr Nephrol 2012;27:2099-106.

31. Li Z, Liu X, Liu S, et al. Urinary heme oxygenase-1 in children with congenital hydronephrosis due to ureteropelvic junction obstruction. Biomarkers 2012;17:471-6.

32. Wasilewska A, Taranta-Janusz K, Dębek W, et al. KIM1 and NGAL: new markers of obstructive nephropathy. Pediatr Nephrol 2011;26:579-86.

33. Bartoli F, Penza R, Aceto G, et al. Urinary epidermal growth factor, monocyte chemotactic protein-1, and $\beta 2$ microglobulin in children with ureteropelvic junction obstruction. J Pediatr Surg 2011;46:530-6.

34. Kajbafzadeh AM, Elmi A, Talab SS, et al. Urinary and serum carbohydrate antigen $19-9$ as a biomarker in ureteropelvic junction obstruction in children. J Urol 2010;183:2353-60.

35. Sager C, Lopez JC, Duran V, et al. Transforming growth factor-beta1 in congenital ureteropelvic junction obstruction: diagnosis and follow-up. Int Braz J Urol 2009;35:315-23; discussion 323-5.

36. Shokeir AA, Taha MA. Role of urinary tubular enzymes in evaluation of children with ureteropelvic junction narrowing under conservative management. Urology 2009;73:1016-20.

37. Taha MA, Shokeir AA, Osman HG, et al. Obstructed versus dilated nonobstructed kidneys in children with 
congenital ureteropelvic junction narrowing: role of urinary tubular enzymes. J Urol 2007;178:640-6.

38. Taha MA, Shokeir AA, Osman HG, et al. Diagnosis of ureteropelvic junction obstruction in children: role of endothelin-1 in voided urine. Urology 2007;69:560-4; discussion 564-5.

39. Taha MA, Shokeir AA, Osman HG, et al. Pelvi-ureteric junction obstruction in children: the role of urinary transforming growth factor-beta and epidermal growth factor. BJU Int 2007;99:899-903.

40. Palmer LS, Maizels M, Kaplan WE, et al. Urine levels of transforming growth factor-beta 1 in children with ureteropelvic junction obstruction. Urology 1997;50:769-73.

Cite this article as: Paraboschi I, Mantica G, Dalton NR, Turner C, Garriboli M. Urinary biomarkers in pelvic-ureteric junction obstruction: a systematic review. Transl Androl Urol 2020;9(2):722-742. doi: 10.21037/tau.2020.01.01
41. El-Sherbiny MT, Mousa OM, Shokeir AA, et al. Role of urinary transforming growth factor-beta1 concentration in the diagnosis of upper urinary tract obstruction in children. J Urol 2002;168:1798-800.

42. Grandaliano G, Gesualdo L, Bartoli F, et al. MCP1 and EGF renal expression and urine excretion in human congenital obstructive nephropathy. Kidney Int 2000;58:182-92.

43. Tekgül S, Dogan HS, Hoebeke P, et al. EAU guidelines on paediatric urology. European Association of Urology 2016:290-323.

44. Biomarkers Definitions Working Group. Biomarkers and surrogate endpoints: preferred definitions and conceptual framework. Clin Pharmacol Ther 2001;69:89-95. 